\title{
BEHAVIOR OF CANONICAL DIVISORS UNDER PURELY INSEPARABLE BASE CHANGES
}

\author{
HIROMU TANAKA
}

\begin{abstract}
Let $k$ be an imperfect field. Let $X$ be a regular variety over $k$ and set $Y$ to be the normalization of $\left(X \otimes_{k} k^{1 / p^{\infty}}\right)_{\text {red. }}$. In this paper, we show that $K_{Y}+C=f^{*} K_{X}$ for some effective divisor $C$ on $Y$. We obtain the following three applications. First, we show that a $K_{X}$-trivial fiber space with non-normal fibers is uniruled. Second, we prove that general fibers of Mori fiber spaces are rationally chain connected. Third, we obtain a weakening of the cone theorem for surfaces and threefolds defined over an imperfect field.
\end{abstract}

\section{Contents}

1. Introduction

2. Preliminaries

2.1. Notation

2.2. Basic properties of purely inseparable base changes

2.3. Known results on dualizing sheaf

3. Field theoretic version of the main theorem

4. Main theorem: canonical divisors and purely inseparable base changes

5. Uniruledness of bad $K_{X}$-trivial fibrations

13

6. RCC-ness of general fibers of Mori fiber spaces 20

7. Cone theorem for surfaces and threefolds 22

Appendix A. MRCC fibrations and base changes

References

\section{INTRODUCTION}

Let $k$ be a field of characteristic $p>0$. Let $X$ be a proper regular variety over $k$. The main interest of this paper is to study a relation between the canonical divisor $K_{X}$ and purely inseparable base changes.

2010 Mathematics Subject Classification. 14E30, 14M22.

Key words and phrases. uniruled, Mori fiber space, cone theorem. 
More precisely, we would like to reduce some problems to the ones for varieties over algebraically closed fields or perfect fields. However, the base change $X \otimes_{k} k^{p^{-\infty}}$, where $k^{p^{-\infty}}:=\bigcup_{e \geq 0} k^{p^{-e}}$, is not normal in general (even not reduced). By taking the normalization $Y$ of the reduced structure $\left(X \otimes_{k} k^{p^{-\infty}}\right)_{\text {red }}$, it is natural to consider the relation between $K_{X}$ and $K_{Y}$. In this paper, we show the following theorem.

Theorem 1.1 (Main theorem, Theorem 4.2). Let $k$ be a field of characteristic $p>0$. Let $X$ be a regular variety over $k$ such that $k$ is algebraically closed in $K(X)$. Set $Y$ to be the normalization of $\left(X \otimes_{k}\right.$ $\left.k^{p^{-\infty}}\right)_{\text {red }}$ and let $Y_{\text {reg }}$ be the regular locus of $Y$. Let $f: Y \rightarrow X$ be the natural morphism. Then, there exists an effective $\mathbb{Z}$-divisor $C$ on $Y$ such that

$$
\left.\left.\left(\omega_{Y / k^{p^{-\infty}}} \otimes_{\mathcal{O}_{Y}} \mathcal{O}_{Y}(C)\right)\right|_{Y_{\mathrm{reg}}} \simeq f^{*} \omega_{X / k}\right|_{Y_{\mathrm{reg}}}
$$

We have $C=0$ if and only if $X$ is geometrically normal over $k$.

In the following, we consider some applications of Theorem 1.1. We can apply this theorem to $K_{X}$-trivial fibrations with non-normal fibers as follows.

Theorem 1.2 (Theorem 5.5). Let $k$ be an algebraically closed field of characteristic $p>0$. Let $\pi: X \rightarrow S$ be a projective $k$-morphism of normal $k$-varieties such that $\pi_{*} \mathcal{O}_{X}=\mathcal{O}_{S}$. Assume that $K_{X}$ is $\mathbb{Q}$-Cartier and $\pi$-numerically trivial. If the generic fiber of $\pi$ is not geometrically normal, then $X$ is uniruled.

Remark 1.3. If $X$ is a smooth surface and $S$ is a curve in Theorem 1.2 , then $X$ is a quasi-elliptic fibration (cf. Bad01, Section 7]), that is, a general fiber is a cuspidal cubic curve.

In the minimal model theory, a Mori fiber space is one of the central objects. In the following result, we see that its general fibers are rationally chain connected.

Theorem 1.4 (Theorem 6.7). Let $k$ be a field. Let $\pi: X \rightarrow S$ be a projective surjective $k$-morphism of normal $k$-varieties such that $\pi_{*} \mathcal{O}_{X}=\mathcal{O}_{S}$. Assume the following conditions.

(1) $X$ is $\mathbb{Q}$-factorial.

(2) $-K_{X}$ is $\pi$-ample.

(3) $\rho(X / S)=1$.

Then general fibers of $\pi$ are rationally chain connected.

Remark 1.5. If $k$ is an algebraically closed field of characteristic zero and $X$ is $\log$ canonical, then Theorem 1.4 follows from HM07, Corollary 1.5]. 
We obtain the following corollary.

Corollary 1.6 (Corollary 6.5). Let $k$ be a field. Let $X$ be a projective normal variety over $k$. Assume the following conditions.

(1) $X$ is $\mathbb{Q}$-factorial.

(2) $-K_{X}$ is ample.

(3) $\rho(X)=1$.

If $D$ is a numerically trivial Cartier divisor on $X$, then there exists $n \in \mathbb{Z}_{>0}$ such that $\mathcal{O}_{X}(n D) \simeq \mathcal{O}_{X}$.

As other applications, we obtain a weakening of cone theorems for surfaces and threefolds over an arbitrary field of positive characteristic.

Theorem 1.7 (Theorem 7.5). Let $k$ be a field of characteristic $p>0$. Let $X$ be a projective normal surface over $k$ and let $\Delta$ be an effective $\mathbb{R}$-divisor such that $K_{X}+\Delta$ is $\mathbb{R}$-Cartier. Let $A$ be an ample $\mathbb{R}$-Cartier $\mathbb{R}$-divisor. Then, there exist finitely many curves $C_{1}, \cdots, C_{m}$ such that

$$
\overline{N E}(X)=\overline{N E}(X)_{K_{X}+\Delta+A \geq 0}+\sum_{i=1}^{m} \mathbb{R}_{\geq 0}\left[C_{i}\right] \text {. }
$$

Theorem 1.8 (Theorem 7.7). Let $k$ be a field of characteristic $p>0$. Let $X$ be a projective normal $\mathbb{Q}$-factorial threefold over $k$ and let $\Delta$ be an effective $\mathbb{R}$-divisor whose coefficients are at most 1 . If $K_{X}+\Delta$ is not nef, then there exist an ample $\mathbb{Q}$-divisor $A$ and finitely many curves $C_{1}, \cdots, C_{m}$ such that $K_{X}+\Delta+A$ is not nef and that

$$
\overline{N E}(X)=\overline{N E}(X)_{K_{X}+\Delta+A \geq 0}+\sum_{i=1}^{m} \mathbb{R}_{\geq 0}\left[C_{i}\right] \text {. }
$$

Proof of the main theorem: We overview the proof of Theorem 1.1. Let $k$ be a field of characteristic $p>0$. Let $X$ be a regular variety over $k$. Set $Y$ to be the normalization of $\left(X \otimes_{k} k^{p^{-\infty}}\right)$ red. Note that $X \otimes_{k} k^{p^{-\infty}}$ is irreducible because Spec $k^{p^{-\infty}} \rightarrow$ Spec $k$ is a universally homeomorphism. We assume that $X$ is not geometrically reduced, otherwise the proof is easy.

By our assumption, $X \otimes_{k} k^{p^{-\infty}}$ is not reduced. However it is difficult to compare dualizing sheaves of $X \otimes_{k} k^{p^{-\infty}}$ and $\left(X \otimes_{k} k^{p^{-\infty}}\right)_{\text {red }}$. Thus our main idea is to avoid non-reduced schemes. For this, we use the following lemma.

Lemma 1.9 (Lemma 3.3). Let $k$ be a field of characteristic $p>0$. Let $F / k$ and $k^{\prime} / k$ be field extensions. Assume the following two conditions.

(1) $k$ is purely inseparably closed in $F$, that is, if $x \in F$ satisfies $x^{p} \in k$, then $x \in k$. 
(2) The field extension $k^{\prime} / k$ is purely inseparable and $\left[k^{\prime}: k\right]=p$. Then $F \otimes_{k} k^{\prime}$ is a field.

Set $F:=K(X)$. Since $k$ is algebraically closed in $F=K(X)$ by the assumption, we see that $k$ is purely inseparably closed in $F$. Let $k^{\prime} / k$ be an arbitrary purely inseparable extension with $\left[k^{\prime}: k\right]=p$. From a pair $(X, k)$, we construct a "bigger" pair $\left(X_{1}, k_{1}\right)$. By Lemma 1.9, we see that $X \otimes_{k} k^{\prime}$ is an integral scheme but may not be normal. By taking the normalization $X_{1}$ of $X \otimes_{k} k^{\prime}, X_{1}$ is a normal variety over $k^{\prime}$. Let $k_{1}$ be the purely inseparable closure of $k^{\prime}$ in $K\left(X_{1}\right)$. Since we can ignore codimension two closed subsets, we may assume that $X_{1}$ is regular. Then $k_{1}$ is purely inseparably closed in $K\left(X_{1}\right)$. By the inductive argument, we obtain sequences $k=: k_{0} \subsetneq k_{1} \subsetneq k_{2} \subsetneq \cdots$ and $X=: X_{0} \leftarrow X_{1} \leftarrow X_{2} \leftarrow \cdots$.

Let us calculate the relation between dualizing sheaves $\omega_{X / k}$ and $\omega_{X_{1} / k_{1}}$. To obtain $\left(X_{1}, k_{1}\right)$ from $(X, k)$, we apply the following three operations: a base change, normalization, replacing a base field $k^{\prime}$ by $k_{1}$ (enlarging base field). Under these operations, dualizing sheaves can be calculated as follows:

- $\omega_{X \otimes_{k} k^{\prime} / k^{\prime}} \simeq \beta^{*} \omega_{X / k}$ (base change)

- $\omega_{X_{1} / k^{\prime}} \otimes_{\mathcal{O}_{X_{1}}} \mathcal{O}_{X_{1}}\left(C_{1}\right) \simeq \nu^{*} \omega_{X \otimes_{k} k^{\prime} / k^{\prime}}$ (normalization)

- $\omega_{X_{1} / k_{1}} \simeq \omega_{X_{1} / k^{\prime}}$ (enlarging a base field),

where $\beta: X \otimes_{k} k^{\prime} \rightarrow X$ is the projection, $\nu: X_{1} \rightarrow X \otimes_{k} k^{\prime}$ is the normalization and $C_{1}$ is an effective divisor. Summarizing above, we obtain

$$
K_{X_{1}}+C_{1}=g^{*} K_{X}
$$

where $g: X_{1} \rightarrow X$ is the induced morphism.

Therefore, it suffices to find, in advance, a finite purely inseparable extension $k \subset k_{\text {goal }}$ such that $k=: k_{0} \subset k_{1} \subset k_{2} \subset \cdots \subset k_{\text {goal }}$ and that the end result $\left(X_{\text {goal }}, k_{\text {goal }}\right)$ satisfies a good property $\left(X_{\text {goal }}\right.$ is geometrically reduced over $k_{\text {goal }}$ and so on $)$. Such a pair $\left(X_{\text {goal }}, k_{\text {goal }}\right)$ can be found as a finite extension model of the pair $\left(\left(X \times_{k} k^{p^{-\infty}}\right)_{\text {red }}^{N}, k^{p^{-\infty}}\right)$ where $\left(X \times_{k} k^{p^{-\infty}}\right)_{\text {red }}^{N}$ is the normalization of $\left(X \times_{k} k^{p^{-\infty}}\right)_{\text {red }}$.

For more details, see Section 3 and Section 4. In Example 4.3, we give an explicit calculation for the double conic curve.

Related results: As a related topic, there is a classical result called Tate's genus change (cf. Sch09] Tat52]). It compares the genus of a given regular curve over an imperfect field and the one of the normalization of its purely inseparable base change of degree $p$.

In Theorem 1.4, we show that general fibers of a Mori fiber space are rationally chain connected. In KMM92, Kollár-Miyaoka-Mori show 
that every smooth Fano variety, defined over an algebraically closed field of any characteristic, is rationally chain connected. After that, Hacon-McKernan ([HM07]) and [Zha06 prove that log Fano varieties in characteristic zero are rationally connected. In positive characteristic, there are few results around this problem (cf. Getal15]).

In Theorem 1.7 and Theorem [1.8, we establish a cone theorem for surfaces and threefolds over a non-closed field. If $k$ is algebraically closed, then Theorem 1.7 and Theorem 1.8 follows from [Tan14 and [CTX], respectively. If $X$ is a smooth projective geometrically connected variety over a non-closed field, then Mori's cone theorem ([KM98, Theorem 1.24]) is established in Mori's original paper ([Mor82, Ch 2, $\S 3])$. Note that Mori's cone theorem is not known even for regular projective varieties because his bend and break technique depends on the smoothness assumption.

In [CZ, Theorem 2.4], Chen-Zhang consider a behavior of canonical bundles under base changes to attack the Iitaka conjecture in positive characteristic. Their setting differs from our main theorem (Theorem 1.1). They consider a positive dimensional base although we treat the case when the base scheme is a field. On the other hand, they assume that the generic fiber is geometrically reduced, however we do not impose such an assumption.

Acknowledgments. The author would like to thank Professors Paolo Cascini, János Kollár, Joseph Lipman, Chenyang Xu, Lei Zhang for valuable comments and fruitful discussions. The author also thanks to the referee for many constructive suggestions. This work is partially supported by JSPS KAKENHI Grant Number 24224001.

\section{PRELIMINARIES}

2.1. Notation. We will not distinguish the notations invertible sheaves and divisors. We say $L$ is an $\mathbb{R}$-invertible sheaf if $L \in \operatorname{Pic}(X) \otimes_{\mathbb{Z}} \mathbb{R}$. A scheme $X$ is normal if the local ring $\mathcal{O}_{X, x}$ for every point $x \in X$ is an integrally closed integral domain. A scheme $X$ is Gorenstein (resp. Cohen-Macaulay, resp. $S_{2}$ ) if so is the local ring $\mathcal{O}_{X, x}$ for every point $x \in X$. In particular, a Gorenstein scheme is Cohen-Macaulay.

We say $X$ is a variety over a field $k$ (or a $k$-variety) if $X$ is an integral scheme which is separated and of finite type over $k$. Note that, in general, this property is not stable under base changes. We say $X$ is a curve (resp. a surface, resp. a threefold) if $X$ is a variety over $k$ with $\operatorname{dim} X=1$ (resp. $\operatorname{dim} X=2$, resp. $\operatorname{dim} X=3$ ). For a scheme $X$, set $X_{\text {red }}$ to be the reduced scheme whose underlying topological space is equal to $X$. 
Let $\Delta$ be an $\mathbb{R}$-divisor on a normal scheme. We write $\Delta \leq a$ if, for the prime decomposition $\Delta=\sum_{i \in I} \delta_{i} \Delta_{i}, \delta_{i} \leq a$ holds for every $i \in I$.

Let $k$ be a field. Let $X$ be a separated scheme of finite type over $k$ and let $C \hookrightarrow X$ be a closed immersion such that $C$ is a proper curve over $k$. Let $L$ be an invertible sheaf on $X$. We define the intersection number $L \cdot C$ by

$$
L \cdot C=\chi\left(C,\left.L\right|_{C}\right)-\chi\left(C, \mathcal{O}_{C}\right) .
$$

It is well-known that this number is an integer. Let $f: X \rightarrow Y$ be a proper morphism of noetherian schemes, and let $L$ and $M$ be an $\mathbb{R}$ invertible sheaves on $X$. We say $L$ and $M$ are $f$-numerically equivalent, written $L \equiv_{f} M$, if $L \cdot C=M \cdot C$ for every proper curve $C$ on $X$ over a closed point $y \in Y$. We say $L$ is $f$-numerically trivial, written $L \equiv_{f} 0$, if $L$ and $\mathcal{O}_{X}$ are $f$-numerically equivalent. If $Y=$ Spec $k$ for a field $k$, then we merely wright $L \equiv 0$.

We will freely use the notation and terminology in KM98 and Kol13. For the definition of uniruled and rationally chain connected varieties, see [Kol96, Ch IV, Definition 1.1 and Definition 3.2]. For the definition of dualizing sheaves and canonical divisors, see Subsection 2.3 .

For a field $k$ of characteristic $p>0$, we fix an algebraic closure $\bar{k}$ and set $k^{p^{-\infty}}:=\bigcup_{e \geq 0}\left\{a \in \bar{k} \mid a^{p^{e}} \in k\right\}$.

Definition 2.1. Let $A \subset B$ be a ring extension of $\mathbb{F}_{p}$-algebras.

(1) The purely inseparable closure $C$ of $A$ in $B$ is the intermediate ring $A \subset C \subset B$ such that for every $b \in B$ with $b^{p^{e}} \in A$ for some exponent $e \geq 0$, then $b \in C$.

(2) $A$ is purely inseparably closed in $B$ if the purely inseparable closure $C$ of $A$ in $B$ satisfies $A=C$.

Note that $A$ is purely inseparably closed in $B$ if and only if an element $\gamma \in B$ satisfies $\gamma^{p} \in A$, then $\gamma \in A$.

2.2. Basic properties of purely inseparable base changes. In this subsection, we summarize basic properties of purely inseparable base changes. Some of them may be known results.

Lemma 2.2. Let $k$ be a field. Let $X$ be a normal variety over $k$. Then the following assertions hold.

(1) If $X$ is geometrically connected, then $X$ is geometrically irreducible over $k$.

(2) Let $k \subset k^{\prime}$ be a field extension. Then the normalization morphism of $\left(X \otimes_{k} k^{\prime}\right)_{\text {red }}$ is a universal homeomorphism. 
Proof. Note that if $X$ is normal and connected, then $X$ is integral. Thus if $k$ is characteristic zero, then both of the assertions are clear. We assume that $k$ is of characteristic $p>0$.

Assuming (2), we prove (1). We apply (2) for the algebraic closure $k^{\prime}:=\bar{k}$. Then we see that the normalization morphism $\left(X \otimes_{k} \bar{k}\right)_{\text {red }}^{N} \rightarrow$ $\left(X \otimes_{k} \bar{k}\right)_{\text {red }}$ is a universal homeomorphism. Since $\left(X \otimes_{k} \bar{k}\right)_{\text {red }}$ is connected by our assumption, so is $\left(X \otimes_{k} \bar{k}\right)_{\text {red }}^{N}$. Thus $\left(X \otimes_{k} \bar{k}\right)_{\text {red }}^{N}$ is connected and normal, hence it is integral. Therefore $X \otimes_{k} \bar{k}$ is irreducible because the composition morphism

$$
\left(X \otimes_{k} \bar{k}\right)_{\mathrm{red}}^{N} \rightarrow\left(X \otimes_{k} \bar{k}\right)_{\mathrm{red}} \rightarrow X \otimes_{k} \bar{k}
$$

is a universally homeomorphism. This implies (1).

It suffices to show (2). We reduce the proof to the case when $k \subset k^{\prime}$ is a purely inseparable extension. For a field extension $k \subset k^{\prime}$, we obtain the following decomposition

$$
k \subset k_{1} \subset k_{2} \subset k^{\prime},
$$

where $k_{1} / k$ is purely transcendental, $k_{2} / k_{1}$ is algebraic separable, and $k^{\prime} / k_{2}$ is purely inseparable. We can check that $X \times_{k} k_{1}$ is normal and hence $X \times_{k} k_{2}$ is also normal. Therefore, we may assume that $k \subset k^{\prime}$ is purely inseparable.

Set $Y$ to be the normalization of $\left(X \otimes_{k} k^{\prime}\right)_{\text {red. }}$ Then, we obtain the following commutative diagram

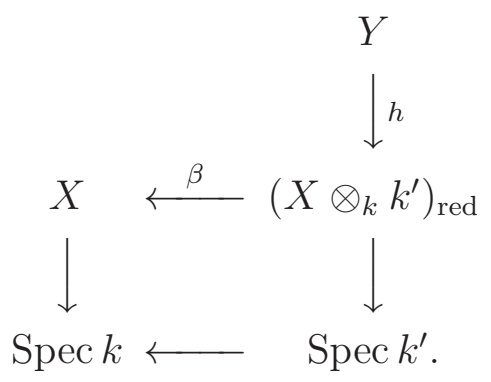

Since $X$ and $Y$ are normal and $Y \rightarrow X$ is an affine integral surjective morphism (i.e. the corresponding ring extensions are integral), $Y$ can be obtained by the integral closure of $X$ in $K(Y)$. Since the field extension $K(Y) / K(X)$ is purely inseparable, the ring extensions

$$
\mathcal{O}_{X}(U) \hookrightarrow \mathcal{O}_{\left(X \otimes_{k} k^{\prime}\right)_{\text {red }}}\left(\beta^{-1}(U)\right) \hookrightarrow \mathcal{O}_{Y}\left(h^{-1} \beta^{-1}(U)\right)
$$

is purely inseparable for every affine open subset $U$ on $X$. Since $\beta$ : $\left(X \otimes_{k} k^{\prime}\right)_{\text {red }} \rightarrow X$ is a universally homeomorphism, the ring extension

$$
\mathcal{O}_{\left(X \otimes_{k} k^{\prime}\right)_{\text {red }}}(V) \hookrightarrow \mathcal{O}_{Y}\left(h^{-1} V\right)
$$


is purely inseparable for every affine open subset $V$ of $\left(X \otimes_{k} k^{\prime}\right)_{\text {red }}$. Therefore, $h$ is a universally homeomorphism.

Positivity of intersection number does not change under base changes.

Lemma 2.3. Let $k \subset k^{\prime}$ be a field extension. Let $C$ be a proper $k$ curve and fix a proper $k^{\prime}$-curve $B$ equipped with a closed immersion $B \hookrightarrow C \otimes_{k} k^{\prime}$. Set $\beta: B \hookrightarrow C \otimes_{k} k^{\prime} \rightarrow C$ to be the composite morphism. Let $L$ be an invertible sheaf on $C$. Then, the following assertions hold.

(1) $L \cdot C>0$ if and only if $\beta^{*} L \cdot B>0$.

(2) $L \cdot C<0$ if and only if $\beta^{*} L \cdot B<0$.

(3) $L \cdot C=0$ if and only if $\beta^{*} L \cdot B=0$.

Proof. (2) follows from (1). (3) holds by (1) and (2). Thus, we only show (1).

By a standard argument, we can assume that $k \subset k^{\prime}$ is a finite extension. Then, $B \rightarrow C$ is a finite surjective $k$-morphism between proper $k$-curves. The assertion holds from the fact that $L$ is ample if and only if $\beta^{*} L$ is ample.

By a purely inseparable base change, Picard numbers do not change.

Proposition 2.4. Let $k$ be a field of characteristic $p>0$.

(1) Let $f: X \rightarrow Y$ be a finite $k$-morphism between proper $k$ schemes. If $f$ is a universal homeomorphism, then $\rho(X)=$ $\rho(Y)$.

(2) Let $k \subset k^{\prime}$ be a (possibly infinite) purely inseparable field extension. Let $X$ be a proper scheme over $k$. Then, $\rho(X)=$ $\rho\left(X \otimes_{k} k^{\prime}\right)$.

(3) Let $X$ be a proper normal variety over $k$. Set $Y$ to be the normalization of $\left(X \otimes_{k} k^{p^{-\infty}}\right)_{\text {red. }}$. Then, $\rho(X)=\rho(Y)$.

Proof. (1) See [Kee99, Lemma 1.4(3)].

(2) Let $\beta: X \otimes_{k} k^{\prime} \rightarrow X$ be the projection. Fix an invertible sheaf $L$ on $X$. By Lemma 2.3, $L \equiv 0$ if and only if $\beta^{*} L \equiv 0$. Thus, we obtain a $\mathbb{Q}$-linear map

$$
\tilde{\beta}:(\operatorname{Pic}(X) / \equiv)_{\mathbb{Q}} \rightarrow\left(\operatorname{Pic}\left(X \otimes_{k} k^{\prime}\right) / \equiv\right)_{\mathbb{Q}}, \quad L \mapsto \beta^{*} L
$$

which is injective, where $M_{\mathbb{Q}}:=M \otimes_{\mathbb{Z}} \mathbb{Q}$ for a $\mathbb{Z}$-module $M$. We show that $\tilde{\beta}$ is surjective. Let $L$ be an invertible sheaf on $X \otimes_{k} k^{\prime}$. Then, we can find an intermediate field $k \subset k_{1} \subset k^{\prime}$ such that $k \subset k_{1}$ is a finite extension and that $L$ is defined over $k_{1}$, that is, there is an invertible sheaf on $L_{1}$ on $X \otimes_{k} k_{1}$ whose pull-back is $L$. By (1), we can find $L_{X} \in \operatorname{Pic}(X)_{\mathbb{Q}}$ such that $\beta_{1}^{*} L_{X} \equiv L$ where $\beta_{1}: X \otimes_{k} k_{1} \rightarrow X$. This implies the surjectivity of $\tilde{\beta}$. 
(3) By Lemma 2.2, $Y \rightarrow X \otimes_{k} k^{p^{-\infty}}$ is a universal homeomorphism. Then, the assertion follows from (1) and (2).

A purely inseparable cover of a $\mathbb{Q}$-factorial variety is $\mathbb{Q}$-factorial.

Lemma 2.5. Let $f: X \rightarrow Y$ be a morphism of noetherian integral normal schemes. If $f$ is homeomorphic and $Y$ is $\mathbb{Q}$-factorial, then $X$ is $\mathbb{Q}$-factorial.

Proof. Let $D_{X}$ be a prime divisor on $X$. Note that prime divisors are irreducible closed subsets of codimension one. Thus $\operatorname{Supp}\left(f\left(D_{X}\right)\right)=: D_{Y}$ is also a prime divisor on $Y$. Since $Y$ is $\mathbb{Q}$-factorial, $m D_{Y}$ is Cartier for some $m \in \mathbb{Z}_{>0}$. Then, $f^{*}\left(m D_{Y}\right)$ is also Cartier and $\operatorname{Supp}\left(f^{*}\left(m D_{Y}\right)\right)=$ $\operatorname{Supp} D_{X}$. Thus, $f^{*}\left(m D_{Y}\right)=n D_{X}$ for some $n \in \mathbb{Z}_{>0}$.

2.3. Known results on dualizing sheaf. We recall the definition of dualizing sheaves and collect some basic properties. For more details, see [Con00, Har66], and [LH09].

For a separated and of finite type morphism of noetherian schemes $f: X \rightarrow Y$, there is a functor

$$
f^{!}: D_{\mathrm{qc}}^{+}(Y) \rightarrow D_{\mathrm{qc}}^{+}(X)
$$

which satisfies the following properties (cf. Har66, Ch. III, Theorem 8.7])

- $(f \circ g)^{!} \simeq g^{!} f^{!}$for two such morphisms $f: X \rightarrow Y$ and $Y \rightarrow Z$.

- If $f$ is a smooth morphism of relative pure dimension $n$, then $f^{!} G^{\bullet} \simeq f^{*}\left(G^{\bullet}\right) \otimes_{\mathcal{O}_{X}}\left(\bigwedge^{n} \Omega_{X / Y}\right)[n]$.

- If $f$ is a finite morphism, then $f^{!} G^{\bullet} \simeq \bar{f}^{*} R \operatorname{Rom}_{\mathcal{O}_{Y}}\left(f_{*} \mathcal{O}_{X}, G^{\bullet}\right)$, where $\bar{f}^{*}$ is the natural exact functor (cf. [Har66, Ch. III, §6])

$$
\bar{f}^{*}: \operatorname{Mod}\left(f_{*} \mathcal{O}_{X}\right) \rightarrow \operatorname{Mod}(X) .
$$

Note that, in general, we need the Nagata compactification to define $f^{!}$.

Definition 2.6. Let $k$ be a field. Let $X$ be a $d$-dimensional separated scheme of finite type over $k$. We set

$$
\omega_{X / k}:=\mathcal{H}^{-d}\left(\alpha^{!} \mathcal{O}_{\text {Spec } k}\right),
$$

where $\alpha: X \rightarrow$ Spec $k$ is the structure morphism.

A dualizing sheaf does not change by enlarging a base field.

Lemma 2.7. Let $k / k_{0}$ be a finite field extension. Let $X$ be a separated scheme of finite type over $k$. Note that $X$ is also of finite type over $k_{0}$. Then, there exists an isomorphism

$$
\omega_{X / k} \simeq \omega_{X / k_{0}}
$$


Proof. Set $\alpha: X \stackrel{\beta}{\rightarrow}$ Spec $k \stackrel{\theta}{\rightarrow}$ Spec $k_{0}$. By $\alpha^{!}=\beta^{!} \theta^{!}$, it suffices to show $\theta^{!} \mathcal{O}_{\text {Spec } k_{0}} \simeq \mathcal{O}_{\text {Spec } k}$. This follows from $\operatorname{Hom}_{k_{0}}\left(k, k_{0}\right) \simeq k$.

Thanks to Lemma 2.7. we can define canonical divisors $K_{X}$ independent of a base field.

Definition 2.8. Let $k$ be a field. If $X$ is a normal variety over $k$, then it is well-known that $\omega_{X / k}$ is a reflexive sheaf. Let $K_{X}$ be a divisor which satisfies

$$
\mathcal{O}_{X}\left(K_{X}\right) \simeq \omega_{X / k}
$$

Such a divisor $K_{X}$ is called canonical divisor. Note that a canonical divisor is determined up to linear equivalence.

We need the following result.

Theorem 2.9. Let $k^{\prime} / k$ be a field extension. Let $X$ be a pure dimensional Cohen-Macaulay separated scheme of finite type over $k$. Let $\beta: X \otimes_{k} k^{\prime} \rightarrow X$ be the projection. Then,

$$
\beta^{*} \omega_{X / k} \simeq \omega_{X \otimes_{k} k^{\prime} / k^{\prime}}
$$

Proof. See, for example, Con00, Theorem 3.6.1] or [LH09, Theorem 4.4.3].

Before stating a result on normalizations, let us recall some notation and basic properties. Let $X$ be a variety over a field $k$ and let $\nu$ : $X^{N} \rightarrow X$ be the normalization. Let $\mathcal{D}$ be the closed subscheme on $X$ defined by the coherent ideal sheaf $\mathcal{H o m}_{\mathcal{O}_{X}}\left(\nu_{*} \mathcal{O}_{X^{N}}, \mathcal{O}_{X}\right) \subset \mathcal{O}_{X}$. Set $\mathcal{C}:=\mathcal{D} \times_{X} X^{N}$. We call $\mathcal{C}$ the conductor scheme of $\nu$. If $X$ is $S_{2}$, then $\mathcal{C}$ is of pure codimension one. Under the normalization, dualizing sheaves are changed as follows.

Proposition 2.10. Let $k$ be a field. Let $X$ be a Gorenstein variety over $k$. Set $\nu: X^{N} \rightarrow X$ to be the normalization and let $\left(X^{N}\right)_{\text {reg }}$ be the regular locus of $X^{N}$. Then,

$$
\left.\left.\left(\omega_{X / k} \otimes_{\mathcal{O}_{X}} \mathcal{O}_{X}(C)\right)\right|_{\left(X^{N}\right)_{\mathrm{reg}}} \simeq\left(\nu^{*} \omega_{X / k}\right)\right|_{\left(X^{N}\right)_{\mathrm{reg}}}
$$

where $C$ is an effective $\mathbb{Z}$-divisor on $X^{N}$ whose support is the same as the support of the conductor scheme $\mathcal{C}$ of $\nu$.

Proof. See [Rei94, Proposition 2.3].

\section{Field theORETIC VERSION OF THE MAIN THEOREM}

The main result of this section is Proposition 3.4, which plays a crucial role in Section 4 (cf. Lemma 4.1). For this, we need the following three lemmas. 
Lemma 3.1. Let $k$ be a field of characteristic $p>0$. Let $F$ be a field containing $k$. If $F$ is geometrically reduced over $k$, then $k$ is purely inseparably closed in $F$.

Proof. The assertion follows from Bou03, Ch. V, §15, 4. Mac Lane's separability criterion, Theorem 2(e)].

Lemma 3.2. Let $k$ be a field and let $F$ be a finitely generated field over $k$. Let $A$ be an arbitrary noetherian k-algebra. Then, $F \otimes_{k} A$ is a noetherian ring.

Proof. We can find a finitely generated $k$-subalgebra $R \subset F$ whose fractional field is $F$. Set $S:=R \backslash\{0\}$ and we see $F=S^{-1} R$. We can check that there exists the following ring isomorphism

$$
F \otimes_{k} A=\left(S^{-1} R\right) \otimes_{k} A \simeq T^{-1}\left(R \otimes_{k} A\right)
$$

where $T:=\{s \otimes 1 \mid s \in S\} \subset R \otimes_{k} A$. By the Hilbert basis theorem, $R \otimes_{k} A$ is a noetherian ring. Therefore, so is $F \otimes_{k} A \simeq T^{-1}\left(R \otimes_{k} A\right)$.

The following lemma is a little bit generalization of [Sch10, Lemma 1.3].

Lemma 3.3. Let $k$ be a field of characteristic $p>0$. Let $F / k$ and $k^{\prime} / k$ be field extensions. Assume the following two conditions.

(1) $k$ is purely inseparably closed in $F$.

(2) The field extension $k^{\prime} / k$ is purely inseparable and $\left[k^{\prime}: k\right]=p$.

Then, $F \otimes_{k} k^{\prime}$ is a field.

Proof. Fix $\alpha \in k^{\prime} \backslash k$ and we see $\beta:=\alpha^{p} \in k$. By (2), we see $k^{\prime}=$ $k[\alpha] \simeq k[t] /\left(t^{p}-\beta\right)$. This implies

$$
F \otimes_{k} k^{\prime} \simeq F[t] /\left(t^{p}-\beta\right) .
$$

It is enough to show that there is no element $\gamma \in F$ such that $\gamma^{p}=\beta$. If not, the field extensions $k \subset k[\gamma] \subset F$ violate the condition (1).

We show the main result in this section.

Proposition 3.4. Let $k$ be a field of characteristic $p>0$. Let $F$ be a finitely generated field over $k$. Then, there exist sequences of field extensions

- $k=: k_{1}^{0} \subset k_{1} \subset k_{2}^{0} \subset k_{2} \subset \cdots \subset k_{n}^{0} \subset k_{n} \subset k^{p^{-\infty}}$, and

- $F=: F_{1} \subset F_{2} \subset \cdots \subset F_{n} \subset\left(F \otimes_{k} k^{p^{-\infty}}\right)_{\text {red }}$,

which satisfy the following properties.

(1) The field extension $k_{n} / k$ is a finite purely inseparable extension.

(2) The field extension $F_{n} / F$ is a finite purely inseparable extension. 
(3) For every $i$, we obtain $k_{i}^{0} \subset k_{i} \subset F_{i}$ and $k_{i}$ is the purely inseparable closure of $k_{i}^{0}$ in $F_{i}$.

(4) For every $i,\left[k_{i+1}^{0}: k_{i}\right]=p$.

(5) For every $i, F_{i+1} \simeq F_{i} \otimes_{k_{i}} k_{i+1}^{0}$.

(6) $F_{n}$ is geometrically reduced over $k_{n}$.

(7) The canonical map $\theta: F_{n} \otimes_{k_{n}} k^{p^{-\infty}} \rightarrow\left(F \otimes_{k} k^{p^{-\infty}}\right)_{\text {red }}$ is bijective.

Proof. For a ring $R$, set $\mathrm{Nil}_{R}$ to be its nilradical. Consider the ring $F \otimes_{k} k^{p^{-\infty}}$. This ring is a noetherian ring by Lemma 3.2. Thus, its nilradical $\mathrm{Nil}_{F \otimes_{k} k^{p^{-\infty}}}$ is finitely generated. Then we can find a finite purely inseparable extension $k_{\text {goal }} / k$ with $k_{\text {goal }} \subset k^{p^{-\infty}}$ such that

$$
\mathrm{Nil}_{F \otimes_{k} k_{\text {goal }}} \cdot\left(F \otimes_{k} k^{p^{-\infty}}\right)=\mathrm{Nil}_{F \otimes_{k} k^{p^{-\infty}}} .
$$

Therefore, we obtain

$$
\begin{aligned}
\left(F \otimes_{k} k_{\text {goal }}\right)_{\text {red }} \otimes_{k_{\text {goal }}} k^{p^{-\infty}} & =\left(\left(F \otimes_{k} k_{\text {goal }}\right) / \mathrm{Nil}_{F \otimes_{k} k_{\text {goal }}}\right) \otimes_{k_{\text {goal }}} k^{p^{-\infty}} \\
& =\left(F \otimes_{k} k^{p^{-\infty}}\right) /\left(\operatorname{Nil}_{F \otimes_{k} k_{\text {goal }}} \cdot\left(F \otimes_{k} k^{p^{-\infty}}\right)\right) \\
& =\left(F \otimes_{k} k^{p^{-\infty}}\right) / \mathrm{Nil}_{F \otimes_{k} k^{p^{-\infty}}} \\
& =\left(F \otimes_{k} k^{p^{-\infty}}\right)_{\text {red }} .
\end{aligned}
$$

In particular,

$$
F_{\text {goal }}:=\left(F \otimes_{k} k_{\text {goal }}\right)_{\text {red }}
$$

is geometrically reduced over $k_{\text {goal }}$. Note that $\left(F \otimes_{k} k^{p^{-\infty}}\right)_{\text {red }}$ and $F_{\text {goal }}$ are fields. We see

$$
k_{\text {goal }} \subset k^{p^{-\infty}}, F_{\text {goal }} \subset\left(F \otimes_{k} k^{p^{-\infty}}\right)_{\text {red }}
$$

and

$$
F_{\text {goal }} \otimes_{k_{\text {goal }}} k^{p^{-\infty}} \simeq\left(F \otimes_{k} k^{p^{-\infty}}\right)_{\text {red }}
$$

We see that $k_{\text {goal }}$ is purely inseparably closed in $F_{\text {goal }}$ by Lemma 3.1. In the following argument, we inductively construct two increasing sequences of subfields of $k_{\text {goal }}$ and of $F_{\text {goal }}$, respectively.

Fix an integer $j \geq 1$ and we assume that we have already constructed two sequences of fields

$$
\begin{gathered}
k_{1}^{0} \subset k_{1} \subset k_{2}^{0} \subset k_{2} \subset \cdots \subset k_{j}^{0} \subset k_{j} \subset k_{\text {goal }} . \\
F_{1} \subset F_{2} \subset \cdots \subset F_{j} \subset F_{\text {goal }}
\end{gathered}
$$

such that, for each $1 \leq i \leq j$, the following properties $(1)_{i}-(5)_{i}$ hold.

$(1)_{i}$ The field extension $k_{i} / k$ is a finite purely inseparable extension.

$(2)_{i}$ The field extension $F_{i} / F$ is a finite purely inseparable extension.

$(3)_{i} k_{i}^{0} \subset k_{i} \subset F_{i}$ and $k_{i}$ is the purely inseparable closure of $k_{i}^{0}$ in $F_{i}$. 
$(4)_{i}\left[k_{i}^{0}: k_{i-1}\right]=p$.

$(5)_{i} F_{i} \simeq F_{i-1} \otimes_{k_{i-1}} k_{i}^{0}$.

Assuming $k_{j} \neq k_{\text {goal }}$, we construct $k_{j+1}^{0}, k_{j+1}$ and $F_{j+1}$ which satisfy

$$
\begin{gathered}
k_{j} \subset k_{j+1}^{0} \subset k_{j+1} \subset k_{\text {goal }} \\
F_{j} \subset F_{j+1} \subset F_{\text {goal }}
\end{gathered}
$$

and the properties $(1)_{j+1^{-}}-(5)_{j+1}$. Since $k_{j} \neq k_{\text {goal }}$, there is an intermediate field $k_{j} \subsetneq k_{j+1}^{0} \subset k_{\text {goal }}$ such that $\left[k_{j+1}^{0}: k_{j}\right]=p$. This implies $(4)_{j+1}$. Set $F_{j+1}:=F_{j} \otimes_{k_{j}} k_{j+1}^{0}$ and $(5)_{j+1}$ holds. Thanks to $(3)_{j}$ and $(4)_{j+1}$, we can apply Lemma 3.3 to $F_{j+1} \simeq F_{j} \otimes_{k_{j}} k_{j+1}^{0}$, and see that $F_{j+1}$ is a field. Let $k_{j+1}$ be the purely inseparable closure of $k_{j+1}^{0}$ in $F_{j+1}$. Then $(3)_{j+1}$ holds. We show $k_{j+1} \subset k_{\text {goal }}$ and $F_{j+1} \subset F_{\text {goal }}$. Note that these imply $(1)_{j+1}$ and $(2)_{j+1}$, respectively. Since $F_{j}, k_{j}$ and $k_{j+1}^{0}$ are contained in $F_{\text {goal }}$, we obtain a natural ring homomorphism

$$
F_{j+1}=F_{j} \otimes_{k_{j}} k_{j+1}^{0} \rightarrow F_{\text {goal }} .
$$

Since $F_{j+1}$ and $F_{\text {goal }}$ are fields, this ring homomorphism is automatically injective. Thus, we obtain $F_{j+1} \subset F_{\text {goal }}$ by replacing $F_{j+1}$ with the image of the injection $F_{j+1} \rightarrow F_{\text {goal }}$. Then we obtain $k_{j+1} \subset k_{\text {goal }}$ because $k_{j+1}^{0} \subset k_{\text {goal }}$ and $k_{\text {goal }}$ is purely inseparably closed in $F_{\text {goal }}$.

By the above inductive construction, we obtain two sequences

$$
\begin{gathered}
k_{1}^{0} \subset k_{1} \subset k_{2}^{0} \subset k_{2} \subset \cdots \subset k_{n}^{0} \subset k_{n}=k_{\text {goal }} \\
F_{1} \subset F_{2} \subset \cdots \subset F_{n} \subset F_{\text {goal }}
\end{gathered}
$$

which satisfy (1)-(5) in the proposition and the following two conditions:

$(6)^{\prime} F_{\text {goal }}$ is geometrically reduced over $k_{n}=k_{\text {goal }}$.

$(7)^{\prime} F_{\text {goal }} \otimes_{k_{\text {goal }}} k^{1 / p^{\infty}} \simeq\left(F \otimes_{k} k^{p^{-\infty}}\right)_{\text {red }}$.

Thus, to show (6) and (7), we prove $F_{n}=F_{\text {goal }}$. For this, it is enough to prove $F_{n} \supset F_{\text {goal }}$. By $F_{\text {goal }}=\left(F_{1} \otimes_{k_{1}} k_{\text {goal }}\right)_{\text {red }}$, it suffices to show that $F_{n} \supset F_{1}$ and $F_{n} \supset k_{\text {goal }}$, which is obvious.

\section{MAIN THEOREM: CANONICAL DIVISORS AND PURELY INSEPARABLE BASE CHANGES}

In this section, we show the main theorem of this paper (Theorem 4.2). We start with a lemma which almost follows from Proposition 3.4

Lemma 4.1. Let $k$ be a field of characteristic $p>0$. Let $X$ be a normal variety over $k$. Then, there exist sequences

$$
\text { - } k=: k_{1}^{0} \subset k_{1} \subset k_{2}^{0} \subset k_{2} \subset \cdots \subset k_{n}^{0} \subset k_{n} \subset k^{p^{-\infty}},
$$


- $X=: X_{1} \leftarrow X_{2} \leftarrow \cdots \leftarrow X_{n} \leftarrow\left(\left(X \otimes_{k} k^{p^{-\infty}}\right)_{\mathrm{red}}\right)^{N}$, and

- $X_{i} \rightarrow \operatorname{Spec} k_{i} \rightarrow \operatorname{Spec} k_{i}^{0}$,

where $\left(\left(X \times_{k} k^{p^{-\infty}}\right)_{\text {red }}\right)^{N}$ is the normalization of $\left(X \otimes_{k} k^{1 / p^{\infty}}\right)_{\text {red }}$ which satisfy the following properties.

(1) The field extension $k_{n} / k$ is a finite purely inseparable extension.

(2) For every $i, X_{i}$ is a normal variety over $k_{i}$, and the morphism $X_{i} \rightarrow X_{i-1}$ is a finite surjective purely inseparable morphism.

(3) For every $i, k_{i}$ is the purely inseparable closure of $k_{i}^{0}$ in $K\left(X_{i}\right)$.

(4) For every $i,\left[k_{i+1}^{0}: k_{i}\right]=p$.

(5) For every $i, X_{i-1} \otimes_{k_{i-1}} k_{i}^{0}$ is integral and $X_{i}$ is the normalization of $X_{i-1} \otimes_{k_{i-1}} k_{i}^{0}$.

(6) $X_{n}$ is geometrically reduced over $k_{n}$.

(7) The induced morphism $\left(\left(X \otimes_{k} k^{p^{-\infty}}\right)_{\mathrm{red}}\right)^{N} \rightarrow X_{n} \otimes_{k_{n}} k^{p^{-\infty}}$ is the normalization of $X_{n} \otimes_{k_{n}} k^{p^{-\infty}}$.

Proof. Set $F:=K(X)$. Then, we can apply Proposition 3.4 and we obtain sequences of field extensions

- $k=: k_{1}^{0} \subset k_{1} \subset k_{2}^{0} \subset k_{2} \subset \cdots \subset k_{n}^{0} \subset k_{n} \subset k^{p^{-\infty}}$, and

- $F=: F_{1} \subset F_{2} \subset \cdots \subset F_{n} \subset\left(F \otimes_{k} k^{p^{-\infty}}\right)_{\text {red }}$,

which satisfy the properties (1)-(7) in Proposition 3.4. We construct a sequence

$$
X=: X_{1} \leftarrow X_{2} \leftarrow \cdots \leftarrow X_{n}
$$

which has a morphism $X_{i} \rightarrow$ Spec $k_{i}$ for every $i$ and satisfies properties (1)-(7). Since (1) and (4) in Proposition 3.4 imply (1) and (4) in the lemma respectively, it suffices to show (2), (3), (5), (6), and (7) in the lemma. For this, we inductively construct a normal $k_{i}$-variety $X_{i}$ with $K\left(X_{i}\right)=F_{i}$.

Set $X=: X_{1}$. Since $X_{1}$ is normal, the structure morphism $X_{1} \rightarrow$ Spec $k_{1}^{0}$ factors through Spec $k_{1}$. Therefore, we can take the fiber product $X_{1} \otimes_{k_{1}} k_{2}^{0}$. The ring corresponding to an open affine subscheme of $X_{1} \otimes_{k_{1}} k_{2}^{0}$ is contained in a field $F_{2}=F_{1} \otimes_{k_{1}} k_{2}^{0}$. Thus, $X_{1} \otimes_{k_{1}} k_{2}^{0}$ is an integral scheme. Take the normalization $X_{2}$ of $X_{1} \otimes_{k_{1}} k_{2}^{0}$ and we see $K\left(X_{2}\right)=F_{2}$. We obtain $X_{2} \rightarrow$ Spec $k_{2} \rightarrow$ Spec $k_{2}^{0}$ and, for $i=2$, properties (2), (3), (5) in the lemma follow from (2), (3), (5) in Proposition 3.4. By the same argument, we can construct $X_{1}, X_{2}, X_{3}, \cdots, X_{n}$ which satisfy (1)-(5) and $K\left(X_{i}\right)=F_{i}$. Moreover, (6) and (7) in the lemma follow from (6) and (7) in Proposition 3.4, respectively. We are done.

We show the main theorem of this paper. 
Theorem 4.2. Let $k$ be a field of characteristic $p>0$. Let $X$ be a normal variety over $k$. Set $Y$ to be the normalization of $\left(X \otimes_{k} k^{p^{-\infty}}\right)_{\text {red }}$ and let $f: Y \rightarrow X$ be the induced affine morphism. Let $X_{\mathrm{reg}}$ and $Y_{\mathrm{reg}}$ be the regular loci of $X$ and $Y$, respectively. Then, there exists an effective $\mathbb{Z}$-divisor $C$ on $Y$ such that

$$
\left.\left.\left(\omega_{Y / k^{p}} \otimes_{\mathcal{O}_{Y}} \mathcal{O}_{Y}(C)\right)\right|_{Y_{\mathrm{reg}}} \simeq f^{*}\left(\left.\omega_{X / k}\right|_{X_{\mathrm{reg}}}\right)\right|_{Y_{\mathrm{reg}}} .
$$

Moreover, $C$ can be chosen to be nonzero if one of the following conditions holds.

(a) $X$ is geometrically reduced but not geometrically normal over $k$.

(b) $X$ is not geometrically reduced and $k$ is algebraically closed in $K(X)$.

Proof. We divide the proof into three steps.

Step 1: The assertion in the theorem holds under the condition (a).

Proof of Step 1. Set

$$
f: Y \stackrel{\nu}{\rightarrow} X \otimes_{k} k^{p^{-\infty}} \stackrel{\beta}{\rightarrow} X,
$$

where $\nu$ is the normalization. Since we can omit codimension two closed subsets, we may assume that $X$ and $Y$ are regular. Then $X \otimes_{k} k^{p^{-\infty}}$ is Gorenstein and we obtain

$$
\omega_{X \otimes_{k} k^{p^{-\infty}} / k^{p^{-\infty}}} \simeq \beta^{*} \omega_{X / k}
$$

by Theorem 2.9. Moreover, by Proposition 2.10, we obtain

$$
\omega_{Y / k^{p^{-}}} \otimes_{\mathcal{O}_{Y}} \mathcal{O}_{Y}(C) \simeq \nu^{*} \omega_{X \times_{k} k^{p^{-\infty}} / k^{p^{-\infty}}}
$$

where $C$ is an effective $\mathbb{Z}$-divisor whose support is equal to the conductor of $\nu$.

We show $C \neq 0$. It suffices to show that $X \otimes_{k} k^{p^{-\infty}}$ is not regular in codimension one. Assume that $X \otimes_{k} k^{p^{-\infty}}$ is regular and let us derive a contradiction. Since $X$ is $S_{2}$, so is $X \otimes_{k} k^{p^{-\infty}}$. Then, by Serre's criterion for normality, $X \otimes_{k} k^{p^{-\infty}}$ is normal. This is impossible because $X$ is not geometrically normal over $k$.

Step 2: There exists an effective $\mathbb{Z}$-divisor $C$ on $Y$ such that

$$
\left.\left.\left(\omega_{Y / k^{p^{-\infty}}} \otimes_{\mathcal{O}_{Y}} \mathcal{O}_{Y}(C)\right)\right|_{Y_{\mathrm{reg}}} \simeq f^{*}\left(\left.\omega_{X / k}\right|_{X_{\mathrm{reg}}}\right)\right|_{Y_{\mathrm{reg}}} .
$$

Proof of Step 2. We apply Proposition 4.1 and obtain sequences

- $k=: k_{1}^{0} \subset k_{1} \subset k_{2}^{0} \subset k_{2} \subset \cdots \subset k_{n}^{0} \subset k_{n} \subset k^{p^{-\infty}}$,

- $X=: X_{1} \leftarrow X_{2} \leftarrow \cdots \leftarrow X_{n} \leftarrow Y$, and

- $X_{i} \rightarrow \operatorname{Spec} k_{i} \rightarrow \operatorname{Spec} k_{i}^{0}$, 
which satisfies the properties (1)-(7) in Proposition 4.1.

From $X_{i} \rightarrow$ Spec $k_{i}$, the morphism $X_{i+1} \rightarrow$ Spec $k_{i+1}$ is obtained by the following three steps.

$(\alpha)$ Take the base change $X_{i} \otimes_{k_{i}} k_{i+1}^{0}$ where $X_{i} \otimes_{k_{i}} k_{i+1}^{0}$ is integral.

( $\beta)$ Take the normalization $X_{i+1}$ of $X_{i} \otimes_{k_{i}} k_{i+1}^{0}$.

$(\gamma)$ Let $k_{i+1}$ be the purely inseparable closure of $k_{i+1}^{0}$ in $K\left(X_{i+1}\right)$. Let $\beta_{i}: X_{i} \otimes_{k_{i}} k_{i+1}^{0} \rightarrow X_{i}$ be the projection and let $\nu_{i}: X_{i+1} \rightarrow$ $X_{i} \otimes_{k_{i}} k_{i+1}^{0}$ be the normalization. Then, we can compare $K_{X_{i}}$ and $K_{X_{i+1}}$ as follows.

$(\alpha) \omega_{X_{i} \otimes_{k_{i}} k_{i+1}^{0} / k_{i+1}^{0}} \simeq \beta_{i}^{*} \omega_{X_{i} / k_{i}}$ holds outside a codimension two closed subset (Theorem 2.9).

( $\beta) \omega_{X_{i+1} / k_{i+1}^{0}} \otimes_{\mathcal{O}_{X_{i+1}}} \mathcal{O}_{X_{i+1}}\left(C_{i}\right) \simeq \nu_{i}^{*} \omega_{X_{i} \otimes_{k_{i}} k_{i+1}^{0} / k_{i+1}^{0}}$ holds outside a codimension two closed subset where the support of $C_{i}$ is equal to the support of the conductor of $\nu_{i}$ (Proposition 2.10).

$(\gamma) \omega_{X_{i+1} / k_{i+1}} \simeq \omega_{X_{i+1} / k_{i+1}^{0}}$ (Lemma 2.7)

Note that, outside a codimension two locus, every $X_{i}$ is regular and every $X_{i} \otimes_{k_{i}} k_{i+1}^{0}$ is Gorenstein.

Therefore, we see that

$$
K_{X^{n}}+C^{\prime}=g^{*} K_{X}
$$

for some effective $\mathbb{Z}$-divisor $C^{\prime}$ where $g: X_{n} \rightarrow X$. Note that $C^{\prime}$ is the sum of the pull-backs of $C_{1}, C_{2}, \cdots, C_{n-1}$. Let $h: Y \rightarrow X_{n}$ be the induced morphism. By (7) in Proposition 4.1 and the proof of the case Step 1, we see $K_{Y}+C^{\prime \prime}=h^{*} K_{X_{n}}$ for some effective $\mathbb{Z}$-divisor $C^{\prime \prime}$. Note that $C^{\prime \prime}$ may be zero. Then we obtain

$$
K_{Y}+C^{\prime \prime}+h^{*} C^{\prime}=h^{*}\left(K_{X_{n}}+C^{\prime}\right)=h^{*}\left(g^{*} K_{X}\right)=f^{*} K_{X} .
$$

Set $C:=C^{\prime \prime}+h^{*} C^{\prime}$.

Step 3: If the condition (b) holds, then we may assume that $C \neq 0$.

Proof of Step 3. We may assume that $X$ is proper, because $X$ is not geometrically reduced over $k$ if and only if $K(X)$ is not geometrically reduced over $k$. Since $k$ is algebraically closed in $K(X)$, we obtain $H^{0}\left(X, \mathcal{O}_{X}\right)=k$.

We apply the same argument as Step 2 and use the same notation as Step 2. To show $C \neq 0$, we prove $C^{\prime} \neq 0$. Assume the contrary, that is, assume $C_{i}=0$ for every $i$. Let us derive a contradiction. The equation $C_{i}=0$ implies that every $X_{i} \otimes_{k_{i}} k_{i+1}^{0}$ is regular in codimension one. Thus, every $X_{i} \otimes_{k_{i}} k_{i+1}^{0}$ is normal. Thus $\nu_{i}$ is an isomorphism and $X_{i+1}=X_{i} \otimes_{k_{i}} k_{i+1}^{0}$. 
We show $H^{0}\left(X_{i}, \mathcal{O}_{X_{i}}\right)=k_{i}^{0}$ by the induction on $i$. When $i=1$, this holds by $H^{0}\left(X, \mathcal{O}_{X}\right)=k$. Fix $i \geq 1$ and assume that $H^{0}\left(X_{i}, \mathcal{O}_{X_{i}}\right)=k_{i}^{0}$ holds for $i$. In particular, we see $H^{0}\left(X_{i}, \mathcal{O}_{X_{i}}\right)=k_{i}=k_{i}^{0}$. By the flat base change theorem, $H^{0}\left(X_{i}, \mathcal{O}_{X_{i}}\right)=k_{i}$ implies $H^{0}\left(X_{i+1}, \mathcal{O}_{X_{i+1}}\right)=$ $k_{i+1}^{0}$. Thus we see $H^{0}\left(X_{i}, \mathcal{O}_{X_{i}}\right)=k_{i}^{0}$ for every $i$.

Since $X_{i}$ is normal, $k_{i}^{0}$ is algebraically closed in $K\left(X_{i}\right)$. This implies $k_{i}^{0}=k_{i}$. Then we see that $X_{n} \simeq X \otimes_{k} k_{n}$ and $X_{n}$ is geometrically reduced over $k_{n}$. Therefore, $X$ is geometrically reduced over $k$, which violates our assumption.

The assertion in the theorem holds.

We give an explicit calculation for the double conic.

Example 4.3. Let $F$ be an algebraically closed field of characteristic two. Set $k:=F(s, t)$ to be the purely transcendental extension of $F$ of degree two. Let

$$
X:=\operatorname{Spec} k[x, y] /\left(s x^{2}+t y^{2}+1\right) .
$$

Since $F[s, t, x, y]\left(s x^{2}+t y^{2}+1\right)$ is smooth over $F$ by the Jacobian criterion for the smoothness, its localization $X$ is a regular curve. Set

$$
k^{\prime}:=k\left(s^{1 / 2}\right)=F\left(s^{1 / 2}, t\right) .
$$

Take the base change

$$
\begin{aligned}
X \otimes_{k} k^{\prime} & =\operatorname{Spec} k^{\prime}[x, y] /\left(s x^{2}+t y^{2}+1\right) \\
& \simeq \operatorname{Spec} k^{\prime}[x, y] /\left(x^{2}+t y^{2}+1\right) \\
& \simeq \operatorname{Spec} k^{\prime}[x, y] /\left(x^{2}+t y^{2}\right) .
\end{aligned}
$$

Note that $X \otimes_{k} k^{\prime}$ is integral but not normal because the origin is a unique non-regular point. Taking the blowup at the origin, we see that the normalization $X_{1}$ of $X \otimes_{k} k^{\prime}$ is written by

$$
X_{1}=\operatorname{Spec} k^{\prime}[x, y] /\left(x^{2}+t\right) \simeq \operatorname{Spec} k^{\prime}\left(t^{1 / 2}\right)[y] \simeq \mathbb{A}_{k^{\prime}\left(t^{1 / 2}\right)}^{1} .
$$

We set $k_{1}:=k^{\prime}\left(t^{1 / 2}\right)=F\left(s^{1 / 2}, t^{1 / 2}\right)$. Therefore, $X_{1}$ is geometrically reduced over $k_{1}$.

In Theorem 4.2, the scheme $\left(X \otimes_{k} k^{1 / p^{\infty}}\right)_{\text {red }}$ is not changed by replacing a larger base field $k^{\prime}$ which is purely inseparable over $k$.

Lemma 4.4. Let $k$ be a field of characteristic $p>0$ and let $k^{\prime} / k$ be a finite purely inseparable extension. Let $X$ be a scheme of finite type over $k^{\prime}$. Then, the natural morphism

$$
\theta:\left(X \otimes_{k^{\prime}} k^{\prime p^{-\infty}}\right)_{\text {red }} \rightarrow\left(X \otimes_{k} k^{p^{-\infty}}\right)_{\text {red }} .
$$


is an isomorphism.

Proof. Since $k^{p^{-\infty}}=k^{p^{-\infty}}$, the assertion follows from the fact that $X \otimes_{k^{\prime}} k^{p^{-\infty}} \rightarrow X \otimes_{k} k^{p^{-\infty}}$ is a bijective closed immersion.

\section{UNIRULEDNESS OF BAD $K_{X}$-TRIVIAL FIBRATIONS}

In this section, we show Theorem 5.5. We recall the definition of uniruled varieties.

Definition 5.1 (Ch IV, Definition 1.1 of [Kol96]). Let $k$ be a field. Let $X$ be a variety over $k$. We say $X$ is uniruled if there exist a variety $Y$ of $\operatorname{dim} Y=\operatorname{dim} X-1$ and a dominant rational map

$$
Y \times{ }_{k} \mathbb{P}_{k}^{1} \rightarrow X
$$

For a separated scheme $X$ of finite type over $k, X$ is uniruled if and only if every irreducible component of $X_{\text {red }}$ is uniruled.

The following lemma is fundamental.

Lemma 5.2. Let $k \subset k^{\prime}$ be a field extension. Let $X$ be a normal geometrically connected variety over $k$. Then, $X$ is uniruled if and only if the normalization $Y$ of $\left(X \otimes_{k} k^{\prime}\right)_{\text {red }}$ is uniruled.

Proof. If $k$ is characteristic zero, then the assertion follows from [Kol96, Ch IV, Proposition 1.3]. Assume that $k$ is characteristic $p>0$. Again, by [Kol96, Ch IV, Proposition 1.3], $X$ is uniruled if and only if $X \otimes_{k} k^{\prime}$ is uniruled. By Definition 5.1, $X \otimes_{k} k^{\prime}$ is uniruled if and only if $\left(X \otimes_{k} k^{\prime}\right)_{\text {red }}$ is uniruled. Since the normalization $Y=\left(X \otimes_{k} k^{\prime}\right)_{\mathrm{red}}^{N} \rightarrow\left(X \otimes_{k} k^{\prime}\right)_{\mathrm{red}}$ is a universally homeomorphism by Lemma 2.2(2), this factors through an iterated Frobenius

$$
F^{e}:\left(X \otimes_{k} k^{\prime}\right)_{\mathrm{red}} \rightarrow\left(X \otimes_{k} k^{\prime}\right)_{\mathrm{red}} .
$$

Thus $\left(X \otimes_{k} k^{\prime}\right)_{\text {red }}^{N}$ is uniruled if and only if $\left(X \otimes_{k} k^{\prime}\right)_{\text {red }}$ is uniruled.

Thanks to the bend and break technique, we obtain a criterion for uniruledness.

Lemma 5.3. Let $k$ be a perfect field of characteristic $p>0$. Let $X$ be a projective normal variety over $k$. Assume that the equation $-K_{X}=N+E$ holds as Weil $\mathbb{R}$-divisors where $N$ is a nef $\mathbb{R}$-Cartier $\mathbb{R}$-divisor and $E$ is an effective $\mathbb{R}$-divisor. If $E \neq 0$ or $N \not \equiv 0$, then $X$ is uniruled.

Proof. We can replace $k$ by its algebraic closure $\bar{k}$. Indeed, the pullbacks of $N$ and $E$ by $X \otimes_{k} \bar{k} \rightarrow X$ are nef and effective, respectively. Thus we can assume that $k$ is algebraically closed. Then the assertion follows from the same proof as [Kol96, Ch IV, Corollary 1.14]. 
The following theorem holds only in positive characteristic because a normal variety over a field of characteristic zero is geometrically normal.

Theorem 5.4. Let $k$ be a field of characteristic $p>0$. Let $X$ be a projective normal variety over $k$ such that $H^{0}\left(X, \mathcal{O}_{X}\right)=k$. Assume that $-K_{X}=N+E$ where $N$ is a nef $\mathbb{R}$-Cartier $\mathbb{R}$-divisor and $E$ is an effective $\mathbb{R}$-divisor. If $X$ is not geometrically normal over $k$, then $X$ is uniruled.

Proof. Let $Y$ be the normalization of $\left(X \otimes_{k} k^{p^{-\infty}}\right)_{\text {red }}$ and let $f: Y \rightarrow X$ be the induced morphism. Then, by Theorem 4.2, we obtain

$$
K_{Y}+C=f^{*} K_{X}
$$

for some nonzero effective $\mathbb{Z}$-divisor $C$. By Lemma [5.2, it suffices to show that $Y$ is uniruled. Since $C \neq 0$, the assertion holds by Lemma 5.3.

We show the main theorem in this section.

Theorem 5.5. Let $k$ be a field of characteristic $p>0$. Let $\pi: X \rightarrow S$ be a projective surjective $k$-morphism of normal $k$-varieties such that $\pi_{*} \mathcal{O}_{X}=\mathcal{O}_{S}$. Assume that $-K_{X}=N+E$ where $N$ is a $\pi$-nef $\mathbb{R}$-Cartier $\mathbb{R}$-divisor and $E$ is an effective $\mathbb{R}$-divisor. If the generic fiber of $\pi$ is not normal, then $X$ is uniruled.

Proof. Set $X_{K(S)}:=X \times{ }_{S} K(S)$. To apply Theorem 5.4 for $X_{K(S)} \rightarrow$ Spec $K(S)$, we check that the conditions of Theorem 5.4 hold. Since $\operatorname{Spec} K(S) \rightarrow S$ is flat, we obtain $H^{0}\left(X_{K(S)}, \mathcal{O}_{X_{K(S)}}\right)=K(S)$. We obtain $-K_{X_{K(S)}}=\left.N\right|_{X_{K(S)}}+\left.E\right|_{X_{K(S)}}$. We see that $\left.N\right|_{X_{K(S)}}$ is a nef $\mathbb{R}$ Cartier $\mathbb{R}$-divisor and $\left.E\right|_{X_{K(S)}}$ is an effective $\mathbb{R}$-divisor. Then we can apply Theorem 5.4 for $X_{K(S)} \rightarrow$ Spec $K(S)$, and we see that $X_{K(S)}$ is uniruled over $K(S)$. Thus we obtain a dominant rational map

$$
Y \times_{K(S)} \mathbb{P}_{K(S)}^{1} \rightarrow X_{K(S)}
$$

where $Y$ is a $(\operatorname{dim} X-\operatorname{dim} S-1)$-dimensional variety over $K(S)$. By killing denominators, we can find an open affine $k$-variety $\operatorname{Spec} R \subset$ $S$ and a $(\operatorname{dim} X-1)$-dimensional variety $Y_{R}$ over $R$ which satisfies $Y_{R} \otimes_{R} K(S) \simeq Y$, equipped with a dominant rational map

$$
Y_{R} \times_{R} \mathbb{P}_{R}^{1} \rightarrow X_{R}
$$

where $X_{R}:=\pi^{-1}(\operatorname{Spec} R)$. Since $Y_{R} \times_{R} \mathbb{P}_{R}^{1} \simeq Y_{R} \times_{k} \mathbb{P}_{k}^{1}, X$ is uniruled. 


\section{RCC-NESS OF GENERAL FIBERS OF MORI FIBER SPACES}

In this section, we show Theorem 6.7. We recall the definition of RCC schemes and MRCC-fibrations. For basic properties of them, see [Kol96, Ch. IV., Sections 3 and 5].

Definition 6.1 (Ch. IV, Definition 3.2 of [Kol96]). Let $k$ be a field. Let $X$ be a proper scheme over $k$. We say $X$ is rationally chain connected (for short, RCC) if there is a family of proper and connected algebraic curves $g: U \rightarrow Y$ whose geometric fibers have only rational components with cycle morphism $u: U \rightarrow X$ such that the canonical morphism $U \times_{Y} U \rightarrow X \times_{k} X$ is dominant.

Definition 6.2 (Ch. IV, Definition 5.1 of [Kol96]). Let $k$ be a field. Let $X$ be a proper normal variety over $k$.

(1) We say $X \supset X^{0} \stackrel{\pi}{\rightarrow} Z$ is an $R C C$ fibration if $X^{0}$ is a nonempty open subset of $X$ and $\pi: X^{0} \rightarrow Z$ is a proper surjective morphism such that the fibers are RCC schemes and that $\pi_{*} \mathcal{O}_{X_{0}}=\mathcal{O}_{Z}$

(2) We say $X \supset X^{0} \rightarrow Z$ is an MRCC fibration if $X \supset X^{0} \rightarrow Z$ is an RCC fibration and, for every RCC fibration $X \supset X^{1} \rightarrow W$, there exists a dominant rational map $Z \rightarrow W$ such that the induced field extension $K(Z) \supset K(W)$ is commutative with $K(X) \supset K(Z)$ and $K(X) \supset K(W)$.

Remark 6.3. Let $X$ be a proper scheme over a field $k$.

(1) For a field extension $k^{\prime} / k, X$ is RCC if and only if so is $X \otimes_{k} k^{\prime}$ (cf. Proposition A.4).

(2) If $k$ is an uncountable algebraically closed field, then $X$ is RCC if and only if every pair of closed points $x_{1}, x_{2} \in X$ can be connected by a connected chain of rational curves ([Kol96, Ch. IV, Proposition 3.6]).

Fano varieties with $\rho(X)=1$ play a crucial role in the minimal model theory. The following theorem states that such varieties are rationally chain connected.

Theorem 6.4. Let $k$ be a field. Let $X$ be a projective normal geometrically connected variety over $k$. Assume the following conditions.

- $X$ is $\mathbb{Q}$-factorial.

- $-K_{X}$ is ample.

- $\rho(X)=1$.

Then $X$ is rationally chain connected over $k$. 
Proof. We may assume that $H^{0}\left(X, \mathcal{O}_{X}\right)=k$.

We reduce the proof to the case where $k$ is perfect. Set char $k=: p>$ 0 . Let $Y$ be the normalization of $\left(X \otimes_{k} k^{p^{-\infty}}\right)_{\text {red }}$. Then, we see that $Y$ is $\mathbb{Q}$-factorial (Lemma 2.5) and $\rho(Y)=1$ (Lemma 2.4). Moreover, by Theorem 4.2, we see

$$
K_{Y}+C=f^{*} K_{X}
$$

for some effective divisor $C$ on $Y$. Since $\rho(Y)=1, C$ is nef. In particular, $-K_{Y}$ is ample. Thus, by replacing $X$ with $Y$, we may assume that $k$ is perfect.

Set $Z:=X \otimes_{k} \bar{k}$. Note that $-K_{Z}$ is an ample $\mathbb{Q}$-Cartier divisor. Thus $Z$ is uniruled. Therefore, an MRCC fibration of $Z \supset Z^{0} \rightarrow W$ satisfies $\operatorname{dim} W<\operatorname{dim} Z$. By Lemma A.4, an MRCC fibration

$$
X \supset X^{0} \stackrel{\pi}{\rightarrow} V
$$

also satisfies $\operatorname{dim} V<\operatorname{dim} X$. It suffices to show $\operatorname{dim} V=0$. Assume $\operatorname{dim} V>0$ and let us derive a contradiction. Fix a closed point $v \in V$. We can find an effective Cartier divisor $D_{V}$ on $V$ with $v \notin D_{V}$ by shrinking $V$ if necessary. Take a proper curve $C_{X} \subset \pi^{-1}(v)$ and set $D_{X}$ to be the closure of $\pi^{-1}\left(D_{V}\right)$. Then, $D_{X} \cap C_{X}=\emptyset$ and this implies $D_{X} \cdot C_{X}=0$. Note that, since $X$ is $\mathbb{Q}$-factorial, $D_{X}$ is $\mathbb{Q}$-Cartier. This contradicts $\rho(X)=1$.

Corollary 6.5. Let $k$ be a field. Let $X$ be a projective normal variety over $k$. Assume the following conditions.

(1) $X$ is $\mathbb{Q}$-factorial.

(2) $-K_{X}$ is ample.

(3) $\rho(X)=1$.

Then the torsion subgroup of $\operatorname{Pic}(X)$ coincides with the subgroup of numerically trivial classes.

Proof. We may assume that $H^{0}\left(X, \mathcal{O}_{X}\right)=k$. By the same argument as Theorem 6.4, we may assume that $k$ is a perfect field. Take the base change to the algebraic closure $X \otimes_{k} \bar{k}$. By the assumption, $X \otimes_{k} \bar{k}$ is rationally chain connected. Then, the Albanese variety of $X \otimes_{k} \bar{k}$ is one point. Therefore, $D$ is torsion.

The following lemma is useful to compare the total space and the generic fibers.

Lemma 6.6. Let $k$ be a field. Let $\pi: X \rightarrow S$ be a proper surjective $k$-morphism of normal $k$-varieties such that $\pi_{*} \mathcal{O}_{X}=\mathcal{O}_{S}$.

(1) If $X$ is $\mathbb{Q}$-factorial, then the generic fiber $X_{K(S)}$ is also $\mathbb{Q}$ factorial. 
(2) If $X$ is $\mathbb{Q}$-factorial and $\rho(X / S)=1$, then $\rho\left(X_{K(S)}\right)=1$.

Proof. (1) The assertion follows from the fact that $X_{K(S)}$ is locally obtained as a localization of $X$.

(2) Fix a Cartier divisor $D$ on $X_{K(S)}$ and a curve $C$ on $X_{K(S)}$. Assume $D \cdot C=0$. It suffices to show $D \equiv 0$. For this, fix an arbitrary curve $B$ on $X_{K(S)}$ and we prove $D \cdot B=0$. We can find a non-empty open subset $S^{\prime} \subset S$, a Cartier divisor $D_{S^{\prime}}$ on $\pi^{-1}\left(S^{\prime}\right)$ and flat families of curves $C_{S^{\prime}}, B_{S^{\prime}} \subset \pi^{-1}\left(S^{\prime}\right)$ over $S^{\prime}$, whose pull-back and inverse images to $X_{K(S)}$ are $D, C, B$, respectively. Fix a closed point $s \in S^{\prime}$ and let $D_{s}, C_{s}, B_{s}$ be the pull-back and inverse images to $X_{s}$. By the flatness of $C_{S^{\prime}} \rightarrow S^{\prime}$, we obtain

$$
D_{s} \cdot C_{s}=D_{S^{\prime}} \cdot C_{s}=D \cdot C=0 .
$$

Let $D_{S}$ be the closure of $D_{S^{\prime}}$ in $X$. Since $X$ is $\mathbb{Q}$-factorial, $D_{S}$ is $\mathbb{Q}$ Cartier. Then $\rho(X / S)=1$ and $D_{S} \cdot C_{s}=D_{s} \cdot C_{s}=0$ imply $D_{S} \equiv_{\pi} 0$. Therefore, $D_{S} \cdot B_{s}=0$. By the flatness of $B_{S^{\prime}} \rightarrow S^{\prime}$, we see

$$
D \cdot B=D_{S} \cdot B_{s}=0 .
$$

This is what we want to show.

We show the main theorem in this section.

Theorem 6.7. Let $k$ be a field. Let $\pi: X \rightarrow S$ be a projective surjective $k$-morphism of normal $k$-varieties such that $\pi_{*} \mathcal{O}_{X}=\mathcal{O}_{S}$. Assume the following conditions.

(1) $X$ is $\mathbb{Q}$-factorial.

(2) $-K_{X}$ is $\pi$-ample.

(3) $\rho(X / S)=1$.

Then general fibers of $\pi$ are rationally chain connected, i.e. there exists a non-empty open subset $S^{\prime} \subset S$ such that for every point $s^{\prime} \in S^{\prime}$, the fiber $X_{s^{\prime}}$ is rationally chain connected.

Proof. By Kol96, Ch IV, Corollary 3.5], it suffices to show that the generic fiber is rationally chain connected. For this, we check that the generic fiber $X_{K(S)}$ satisfies the assumptions of Theorem 6.4. By Lemma 6.6, $X_{K(S)}$ is $\mathbb{Q}$-factorial and $\rho\left(X_{K(S)}\right)=1$. Since $-K_{X}$ is $\pi$-ample, $-K_{X_{K(S)}}$ is ample.

\section{Cone THEOREM FOR SURFACES AND THREEFOLDS}

In this section, we show Theorem 7.5 and Theorem 7.7 . We recall the definition of nef thresholds. 
Definition 7.1. Let $k$ be a field. Let $X$ be a projective normal variety over $k$. Let $D$ be an $\mathbb{R}$-Cartier $\mathbb{R}$-divisor and let $H$ be an ample $\mathbb{R}$ Cartier $\mathbb{R}$-divisor. We define a nef threshold $a_{H}$ of $D$ with respect to $H$ by

$$
a_{H}:=\inf \left\{a \in \mathbb{R}_{\geq 0} \mid D+a H \text { is nef }\right\} .
$$

Remark 7.2. Let $k \subset k^{\prime}$ be a field extension. Let $X$ be a projective normal variety over $k$. Set $f: Y \rightarrow X$ be the normalization of $\left(X \otimes_{k}\right.$ $\left.k^{\prime}\right)_{\text {red }}$. Let $D$ be an $\mathbb{R}$-Cartier $\mathbb{R}$-divisor and let $H$ be an ample $\mathbb{R}$ Cartier $\mathbb{R}$-divisor. Then, by Lemma 2.3 , the nef threshold of $D$ with respect to $H$ is equal to the nef threshold of $f^{*} D$ with respect to $f^{*} H$.

Recall that for a $K_{X}$-negative extremal ray $R$ of $\overline{N E}(X)$, its length is defined by

$$
\min \left\{-K_{X} \cdot C \mid C \text { is a curve such that }[C] \in R\right\} .
$$

One of the main strategy to show the cone theorem is to find an upper bound of length of extremal rays. However it may be difficult to find such a bound by the following example.

Example 7.3. Fix a positive integer $N \in \mathbb{Z}_{>0}$. Let $k$ be a field such that $[\bar{k}: k]=\infty$. Let $Y:=\mathbb{P}_{k}^{2}$. Then, we can find a closed point $y \in Y$ such that $[k(y): k]>N$. Take the blowup at $y: f: X \rightarrow Y$ and let $C$ be the $f$-exceptional curve. We see $-K_{X} \cdot C>0$. Note that the structure morphism $C \rightarrow$ Spec $k$ factors through $C \rightarrow \operatorname{Spec} k(y) \rightarrow$ Spec $k$. Thus the residue field of every closed point of $C$ contains $k(y)$. Thus, $-K_{X} \cdot C$ is divisible by $[k(y): k]$. This implies $-K_{X} \cdot C>N$.

To summarize, for every positive integer $N \in \mathbb{Z}_{>0}$, there exist a projective regular surface $X$ over $k$ and a curve $C$ on $X$ which satisfy the following properties.

(1) $\mathbb{R}_{\geq 0}[C] \subset \overline{N E}(X)$ is an extremal ray of $\overline{N E}(X)$.

(2) $-\bar{K}_{X} \cdot C>N$.

(3) $C^{2}<0$.

Instead of the boundedness of length of extremal rays, we use the following lemma.

Lemma 7.4. Let $k$ be a field. Let $X$ be a projective normal variety over $k$. Let $\Delta$ be an $\mathbb{R}$-divisor on $X$ such that $K_{X}+\Delta$ is $\mathbb{R}$-Cartier. Let $A$ be an ample $\mathbb{R}$-Cartier $\mathbb{R}$-divisor on $X$. For any ample $\mathbb{R}$-Cartier $\mathbb{R}$-divisor $H$, let $a_{H}$ be the nef threshold of $K_{X}+\Delta+\frac{1}{2} A$ with respect to $H$.

Assume that there exist finitely many curves $C_{1}, \cdots, C_{m}$, such that for any ample $\mathbb{R}$-Cartier $\mathbb{R}$-divisor $H$ on $X$, we have that $\left(K_{X}+\Delta+\right.$ 
$\left.\frac{1}{2} A+a_{H} H\right) \cdot C_{i}=0$ for some $i$. Then

$$
\overline{N E}(X)=\overline{N E}(X)_{K_{X}+\Delta+A \geq 0}+\sum_{i=1}^{m} \mathbb{R}_{\geq 0}\left[C_{i}\right] .
$$

Proof. We can apply the same proof as CTX, Lemma 6.2].

We show a cone theorem for surfaces.

Theorem 7.5. Let $k$ be a field of characteristic $p>0$. Let $X$ be a projective normal surface over $k$ and let $\Delta$ be an effective $\mathbb{R}$-divisor such that $K_{X}+\Delta$ is $\mathbb{R}$-Cartier. Let $A$ be an ample $\mathbb{R}$-Cartier $\mathbb{R}$-divisor.

(1) For any ample $\mathbb{R}$-Cartier $\mathbb{R}$-divisor $H$, let $a_{H}$ be the nef threshold of $K_{X}+\Delta+A$ with respect to $H$. Then, there exist finitely many curves $C_{1}, \cdots, C_{m}$, such that for any ample $\mathbb{R}$-Cartier $\mathbb{R}$-divisor $H$ on $X$, we have that $\left(K_{X}+\Delta+A+a_{H} H\right) \cdot C_{i}=0$ for some $i$.

(2) There exists a finitely many curves $C_{1}, \cdots, C_{r}$ such that

$$
\overline{N E}(X)=\overline{N E}(X)_{K_{X}+\Delta+A \geq 0}+\sum_{i=1}^{r} \mathbb{R}_{\geq 0}\left[C_{i}\right] .
$$

Proof. Since (2) follows from (1) by Lemma 7.4, we prove (1). We may assume $H^{0}\left(X, \mathcal{O}_{X}\right)=k$ by taking the Stein factorization.

Let $\bar{k}$ be the algebraic closure of $k$. Let $Y$ be the normalization of $\left(X \otimes_{k} \bar{k}\right)_{\text {red. }}$ Then, $Y$ is a normal variety over $\bar{k}$. Let $f: Y \rightarrow X$ be the induced morphism. Then, by Theorem 4.2, we obtain

$$
K_{Y}+D=f^{*} K_{X}
$$

for some effective divisor $D$. Thus, we can find an effective $\mathbb{R}$-divisor $\Delta_{Y}$ such that

$$
K_{Y}+\Delta_{Y}=f^{*}\left(K_{X}+\Delta\right)
$$

and that $K_{Y}+\Delta_{Y}$ is $\mathbb{R}$-Cartier. Thus, $\left(\bar{k}, Y, \Delta_{Y}, f^{*} A\right)$ satisfies the same assumptions as $(k, X, \Delta, A)$. Note that, if $k$ is an algebraically closed field, then the assertion follows from [Tan14, Theorem 3.13(2)]. Then, there exist curves $C_{1}^{\prime}, \cdots, C_{m}^{\prime}$ on $Y$ such that for any ample $\mathbb{R}$-Cartier $\mathbb{R}$-divisor $H^{\prime}$ on $Y$, we have that $\left(K_{Y}+\Delta_{Y}+f^{*} A+b_{H^{\prime}} H^{\prime}\right) \cdot C_{i}^{\prime}=0$ for some $i$, where $b_{H^{\prime}}$ is the nef threshold of $K_{Y}+\Delta_{Y}+f^{*} A$ with respect to $H^{\prime}$. We can find curves $C_{1}, \cdots, C_{m}$ on $X$ such that $C_{i}^{\prime} \subset f^{-1}\left(C_{i}\right)$. Take an ample $\mathbb{R}$-Cartier $\mathbb{R}$-divisor $H$ on $X$. We see $a_{H}=b_{f^{*} H}$ (cf. Remark 7.2). Thus we obtain

$$
\left(K_{Y}+\Delta_{Y}+f^{*} A+a_{H} f^{*} H\right) \cdot C_{i}^{\prime}=0
$$


for some $i$. By Lemma 2.3, this implies

$$
\left(K_{X}+\Delta+A+a_{H} H\right) \cdot C_{i}=0 .
$$

By the cone theorem for surfaces (Theorem 7.5 ), we obtain a cone theorem for threefolds with pseudo-effective canonical divisors.

Theorem 7.6. Let $k$ be a field of characteristic $p>0$. Let $X$ be a projective normal $\mathbb{Q}$-factorial threefold and let $\Delta$ be an effective $\mathbb{R}$ divisor. Assume the following two conditions.

- $0 \leq \Delta \leq 1$.

- $K_{X}+\Delta$ is pseudo-effective

Let $A$ be an ample $\mathbb{R}$-divisor.

(1) For any ample $\mathbb{R}$-divisor $H$, let $a_{H}$ be the nef threshold of $K_{X}+$ $\Delta+A$ with respect to $H$. Then, there exist finitely many curves $C_{1}, \ldots, C_{m}$, such that for any ample $\mathbb{R}$-divisor $H$ on $X$, we have that $\left(K_{X}+\Delta+A+a_{H} H\right) \cdot C_{i}=0$ for some $i$.

(2) There exists a finitely many curves $C_{1}, \cdots, C_{r}$ such that

$$
\overline{N E}(X)=\overline{N E}(X)_{K_{X}+\Delta+A \geq 0}+\sum_{i=1}^{r} \mathbb{R}_{\geq 0}\left[C_{i}\right] .
$$

Proof. Since (2) follows from (1) by Lemma 7.4, we prove (1).

Since $A$ is ample, we can assume $0 \leq \Delta<1$ and $K_{X}+\Delta \equiv E$ where $E$ is an effective $\mathbb{R}$-divisor. Set $E:=\sum_{i=1}^{s} e_{i} E_{i}$ to be the prime decomposition with $e_{i}>0$. We define $\lambda_{i} \in \mathbb{R}_{>0}$ by

$$
K_{X}+\Delta+\lambda_{i} E=K_{X}+E_{i}+D_{i}
$$

where $D_{i}$ is an effective $\mathbb{R}$-divisor with $E_{i} \not \subset \operatorname{Supp} D_{i}$. We obtain

$$
\left(1+\lambda_{i}\right)\left(K_{X}+\Delta\right) \equiv K_{X}+\Delta+\lambda_{i} E=K_{X}+E_{i}+D_{i} .
$$

Set $\nu_{i}: E_{i}^{N} \rightarrow E_{i}$ to be the normalization. We define an effective $\mathbb{R}$-divisor $\Delta_{E_{i}^{N}}$ by the adjunction (cf. [Kol13, Proposition 4.5])

$$
\left.\left(K_{X}+E_{i}+D_{i}\right)\right|_{E_{i}^{N}}=K_{E_{i}^{N}}+\Delta_{E_{i}^{N}}
$$

Let $\Gamma_{1}, \cdots, \Gamma_{r}$ be the curves in $X$ such that the support of $\bigcup_{i=1}^{r} \Gamma_{j}$ coincides with the dimension one part of $\operatorname{Ex}\left(\nu_{1}\right) \cup \cdots \cup \operatorname{Ex}\left(\nu_{s}\right)$. Let $b_{1, H}, \cdots, b_{s, H}$ be the nef thresholds of $\left.\left(K_{X}+\Delta+A\right)\right|_{E_{i}^{N}}$ with respect to $\left.H\right|_{E_{i}^{N}}$. We define $\gamma_{j} \in \mathbb{R}$ by $\left(K_{X}+\Delta+A+\gamma_{j} H\right) \cdot \Gamma_{j}=0$.

Set $\mu:=\max \left\{\max _{1 \leq i \leq s}\left\{b_{i, H}\right\}, \max _{1 \leq j \leq r}\left\{\gamma_{j}\right\}\right\}$ and we show that $a_{H}=\mu$. Since $K_{X}+\Delta+A+a_{H} H$ is nef, $\left.\left(K_{X}+\Delta+A+a_{H} H\right)\right|_{E_{i}^{N}}$ is nef for every $i$ and $\left(K_{X}+\Delta+A+a_{H} H\right) \cdot \Gamma_{j} \geq 0$ for every $j$. Thus, we obtain $a_{H} \geq \mu$. We show the inverse inequality $a_{H} \leq \mu$. 
Assume that $\left(K_{X}+\Delta+A+c H\right) \cdot C<0$ for some $c \geq 0$ and curve $C$. We show that one of $\left\{\left.\left(K_{X}+\Delta+A+c H\right)\right|_{E_{i}^{N}}\right\}_{i}$ is not nef or one of $\left\{\left(K_{X}+\Delta+A+c H\right) \cdot \Gamma_{j}\right\}_{j}$ is negative. Thus, we may assume that $\left(K_{X}+\Delta+A+c H\right) \cdot \Gamma_{j} \geq 0$ for every $j$. In particular, $C \neq \Gamma_{j}$ for every $j$. Since

$$
0>\left(K_{X}+\Delta+A+c H\right) \cdot C>\left(\sum_{i=1}^{s} e_{i} E_{i}\right) \cdot C,
$$

we obtain $E_{i} \cdot C<0$, which implies $C \subset E_{i}$. Since $C \neq \Gamma_{j}$, we can take the proper transform $C^{\prime} \subset E_{i}^{N}$ of $C$, which implies

$0>\left(K_{X}+\Delta+A+c H\right) \cdot C=\left.\left(K_{X}+\Delta+A+c H\right)\right|_{E_{i}} \cdot C=\left.\left(K_{X}+\Delta+A+c H\right)\right|_{E_{i}^{N}} \cdot C^{\prime}$.

Thus $\left.\left(K_{X}+\Delta+A+c H\right)\right|_{E_{i}^{N}}$ is not nef and we obtain $a_{H} \leq \mu$.

We have

$\left.\left.\left(K_{X}+\Delta+A+b_{i, H} H\right)\right|_{E_{i}^{N}} \equiv \frac{1}{1+\lambda_{i}}\left(K_{E_{i}^{N}}+\Delta_{E_{i}^{N}}+\left(1+\lambda_{i}\right) A+b_{i, H}\left(1+\lambda_{i}\right) H\right)\right|_{E_{i}^{N}}$.

We apply Theorem 7.5 for $\left(E_{i}^{N}, \Delta_{E_{i}^{N}} ;\left.\left(1+\lambda_{i}\right) A\right|_{E_{i}^{N}},\left(1+\lambda_{i}\right) H\right)$ and obtain curves $D_{1}^{(i)}, \cdots, D_{t_{i}}^{(i)}$ in $E_{i}^{N}$, which satisfy the property Theorem $7.5(1)$.

We show that the curves $\Gamma_{1}, \cdots, \Gamma_{r}$ and $\left\{\nu_{i}\left(D_{1}^{(i)}\right), \cdots, \nu_{i}\left(D_{t_{i}}^{(i)}\right)\right\}_{1 \leq i \leq s}$ satisfy the required properties. Take an ample $\mathbb{R}$-Cartier $\mathbb{R}$-divisor $\bar{H}$. Since $a_{H}=\mu$, we obtain $a_{H}=b_{i, H}$ for some $i$ or $a_{H}=\gamma_{j}$ for some $j$. If $a_{H}=\gamma_{j}$, then there is nothing to show. We can assume $a_{H}=b_{i, H}$ for some $i$. Then, by the choice of $D_{\ell}^{(i)}$, we can find $D_{\ell}^{(i)}$ such that $\left(K_{X}+\Delta+A+b_{i, H} H\right) \cdot D_{\ell}^{(i)}=0$ for some $\ell$. We are done.

By a result of [CTX], we obtain a weak cone theorem for threefolds over an arbitrary field of positive characteristic.

Theorem 7.7. Let $k$ be a field of characteristic $p>0$. Let $X$ be a projective normal $\mathbb{Q}$-factorial threefold over $k$ and let $\Delta$ be an effective $\mathbb{R}$-divisor such that $0 \leq \Delta \leq 1$. If $K_{X}+\Delta$ is not nef, then there exist an ample $\mathbb{Q}$-divisor $A$ and finitely many curves $C_{1}, \cdots, C_{m}$ such that $K_{X}+\Delta+A$ is not nef and that

$$
\overline{N E}(X)=\overline{N E}(X)_{K_{X}+\Delta+A \geq 0}+\sum_{i=1}^{m} \mathbb{R}_{\geq 0}\left[C_{i}\right] .
$$

Proof. Fix an ample $\mathbb{Q}$-divisor $A_{0}$ such that $K_{X}+\Delta+A_{0}$ is not nef. Set $a_{H}$ to be the nef threshold of $K_{X}+\Delta+A_{0}$ with respect to $H$. There are the following two cases.

(1) There exists an ample $\mathbb{R}$-divisor $H$ such that $K_{X}+\Delta+A_{0}+a_{H} H$ is big. 
(2) For every ample $\mathbb{R}$-divisor $H, K_{X}+\Delta+A_{0}+a_{H} H$ is not big.

(1) Assume that there exists an ample $\mathbb{R}$-divisor $H$ such that $K_{X}+$ $\Delta+A_{0}+a_{H} H$ is big. Then, $K_{X}+\Delta+A_{0}+\left(a_{H}-\epsilon\right) H$ is also big but not nef for a small positive real number $\epsilon>0$. Then, by perturbing $A_{0}+\left(a_{H}-\epsilon\right) H$, we can find an ample $\mathbb{Q}$-divisor $A$ such that $K_{X}+\Delta+A$ is big but not nef. Then, the assertion follows from Theorem 7.6 .

(2) In this case, we set $A:=A_{0}$ and we show that $A$ satisfies the required properties. For every ample $\mathbb{R}$-divisor $H, K_{X}+\Delta+A+a_{H} H$ is not big. We may assume that $H^{0}\left(X, \mathcal{O}_{X}\right)=k$. Set $Y$ to be the normalization of $\left(X \times_{k} \bar{k}\right)_{\text {red }}$ where $\bar{k}$ is the algebraic closure of $k$. By Theorem 4.2, we can write $K_{Y}+D=f^{*} K_{X}$ for some effective divisor $D$ where $f: Y \rightarrow X$ is the induced morphism. Then, we obtain $K_{Y}+\Delta_{Y}=f^{*}\left(K_{X}+\Delta\right)$ for some effective $\mathbb{R}$-divisor $\Delta_{Y}$. Then, by CTX, Lemma 5.3], we can find a curve $C^{\prime}$ on $Y$ such that

$$
\left(K_{Y}+\Delta_{Y}+f^{*}\left(A+a_{H} H\right)\right) \cdot C^{\prime}=0
$$

and that

$$
f^{*} A \cdot C^{\prime} \leq-\left(K_{Y}+\Delta_{Y}\right) \cdot C^{\prime} \leq 2 \operatorname{dim} X .
$$

Thus, we can find curves $C_{1}^{\prime}, \cdots, C_{m}^{\prime}$ on $Y$ such that for every ample $\mathbb{R}$-divisor $H$, we obtain

$$
\left(K_{Y}+\Delta_{Y}+f^{*}\left(A+a_{H} H\right)\right) \cdot C_{i}^{\prime}=0
$$

for some $C_{i}^{\prime}$. Let $C_{i}$ be the curve such that $C_{i}^{\prime} \subset f^{-1}\left(C_{i}\right)$. Then, by Lemma 2.3, for every ample $\mathbb{R}$-divisor $H$, we can find $C_{i}$ such that

$$
\left(K_{X}+\Delta+A+a_{H} H\right) \cdot C_{i}=0 .
$$

By Lemma 7.4, we obtain the assertion.

\section{Appendix A. MRCC Fibrations And BASE CHANGES}

The purpose of this section is to show Proposition A.4. This result is intrinsically shown in the proof of [Kol96, Theorem 5.2], i.e. it depends on [Kol96, Theorem 4.17] and by using the property (4.17.2) in [Kol96, Theorem 4.17], we can deduce Proposition A.4. However, we give a proof of it for the sake of completeness. First, we establish two lemmas.

Lemma A.1. Let $k$ be a field. Let

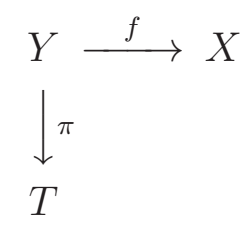

be k-morphisms of $k$-varieties which satisfy the following properties. 
- $X, Y$, and $T$ are normal $k$-varieties.

- $T$ is an affine scheme.

- $\pi$ is a proper surjective morphism such that $\pi_{*} \mathcal{O}_{Y}=\mathcal{O}_{T}$ and $f$ is a finite surjective morphism.

Then $S:=\operatorname{Spec} H^{0}\left(X, \mathcal{O}_{X}\right)$ completes the following commutative diagram

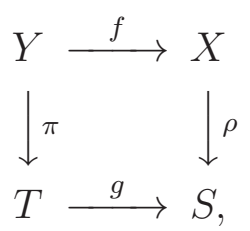

such that $S$ is a normal $k$-variety, where $\rho$ is a proper surjective $k$ morphism such that $\rho_{*} \mathcal{O}_{X}=\mathcal{O}_{S}$ and $g$ is a finite surjective $k$-morphism.

Proof. Fix an affine open cover $X=\bigcup_{i \in I} X_{i}$ and set $Y_{i}:=f^{-1}\left(X_{i}\right)$. Clearly, $S$ satisfies the commutative diagram in the lemma. Note that

$$
\begin{aligned}
& \Gamma\left(S, \mathcal{O}_{S}\right)=\Gamma\left(X, \mathcal{O}_{X}\right)=\bigcap_{x \in X} \mathcal{O}_{X, x}=\bigcap_{i \in I} \Gamma\left(X_{i}, \mathcal{O}_{X}\right) . \\
& \Gamma\left(T, \mathcal{O}_{T}\right)=\Gamma\left(Y, \mathcal{O}_{Y}\right)=\bigcap_{y \in Y} \mathcal{O}_{Y, y}=\bigcap_{i \in I} \Gamma\left(Y_{i}, \mathcal{O}_{Y}\right) .
\end{aligned}
$$

Thus $S$ is an affine integral $k$-scheme. We show that $S$ satisfies the required properties in the lemma. For this, it suffices to show that $g$ is a finite morphism by the Eakin-Nagata theorem. Taking the separable closure of $K(Y) / K(X)$, we may assume that either $g$ is separable or purely inseparable.

Suppose that $K(Y) / K(X)$ is a separable. Let $L \supset K(Y) \supset K(X)$ be the Galois closure and set $G:=\operatorname{Gal}(L / K(X))$. Let $g: Z \rightarrow Y$ be the normalization of $Y$ in $L$ and set $Z_{i}:=g^{-1}\left(Y_{i}\right)$. Since the composite morphism $Z \rightarrow Y \rightarrow T$ is proper, the ring $\Gamma\left(Z, \mathcal{O}_{Z}\right)$ is a finitely generated $k$-algebra. We obtain

$\Gamma\left(S, \mathcal{O}_{S}\right)=\bigcap_{i \in I} \Gamma\left(X_{i}, \mathcal{O}_{X}\right)=\bigcap_{i \in I}\left(\Gamma\left(Z_{i}, \mathcal{O}_{Z}\right)^{G}\right)=\left(\bigcap_{i \in I} \Gamma\left(Z_{i}, \mathcal{O}_{Z}\right)\right)^{G}=\Gamma\left(Z, \mathcal{O}_{Z}\right)^{G}$

Therefore, $\Gamma\left(S, \mathcal{O}_{S}\right)$ is a finitely generated $k$-algebra and $\Gamma\left(Z, \mathcal{O}_{Z}\right)$ is a finitely generated $\Gamma\left(S, \mathcal{O}_{S}\right)$-module, hence so is $\Gamma\left(Y, \mathcal{O}_{Y}\right)=\Gamma\left(T, \mathcal{O}_{T}\right)$. Thus, $g$ is finite.

Therefore, we may assume that $K(Y) / K(X)$ is purely inseparable. We can find $e \in \mathbb{Z}_{>0}$ such that $K(X) \supset K(Y)^{p^{e}}$, in particular, 
$\Gamma\left(X_{i}, \mathcal{O}_{X}\right) \supset \Gamma\left(Y_{i}, \mathcal{O}_{Y}\right)^{p^{e}}$. We have

$$
\begin{gathered}
\Gamma\left(S, \mathcal{O}_{S}\right)=\bigcap_{i \in I} \Gamma\left(X_{i}, \mathcal{O}_{X}\right) \supset k\left[\bigcap_{i \in I}\left(\Gamma\left(Y_{i}, \mathcal{O}_{Y}\right)^{p^{e}}\right)\right] \\
\quad=k\left[\left(\bigcap_{i \in I} \Gamma\left(Y_{i}, \mathcal{O}_{Y}\right)\right)^{p^{e}}\right]=k\left[\Gamma\left(T, \mathcal{O}_{T}\right)^{p^{e}}\right],
\end{gathered}
$$

where $k[A]$ means the minimum $k$-algebra containing $A$. Here $\Gamma\left(T, \mathcal{O}_{T}\right)$ is a finitely generated $k\left[\Gamma\left(T, \mathcal{O}_{T}\right)^{p^{e}}\right]$-module because it is an integral extension. Therefore, $\Gamma\left(T, \mathcal{O}_{T}\right)$ is a finitely generated $\Gamma\left(S, \mathcal{O}_{S}\right)$-module. We are done.

Remark A.2. Lemma A.1 fails when $T$ is not affine. Actually, there is a finite surjective morphism

$$
Y:=\mathbb{P}^{1} \times \mathbb{P}^{1} \rightarrow \mathbb{P}^{2}=: X .
$$

On the other hand, $Y$ has a proper morphism to a curve but $X$ does not.

Lemma A.3. Let $k$ be a field. Let $Y$ be a proper normal $k$-variety. Let $Y^{0} \subset Y$ be a non-empty open subset and let

$$
\pi: Y^{0} \rightarrow Z=\operatorname{Spec} R
$$

be a proper surjective $k$-morphism to an affine $k$-variety $Z$ such that $\pi_{*} \mathcal{O}_{Y^{0}}=\mathcal{O}_{Z}$. We fix an embedding $K(Z) \subset K(Y)$ induced by $\pi$. Then

$$
Y^{0}=\left\{y \in Y \mid R \subset \mathcal{O}_{Y, y}\right\} .
$$

Proof. Set $Y^{1}:=\left\{y \in Y \mid R \subset \mathcal{O}_{Y, y}\right\}$. We show $Y^{0} \subset Y^{1}$. Take $y \in Y^{0}$. Then, we obtain

$$
R=\Gamma\left(Z, \mathcal{O}_{Z}\right)=\Gamma\left(Z, \pi_{*} \mathcal{O}_{Y^{0}}\right)=\Gamma\left(Y^{0}, \mathcal{O}_{Y^{0}}\right) \subset \mathcal{O}_{Y, y}
$$

This implies $y \in Y^{1}$.

We prove the inverse inclusion $Y^{0} \supset Y^{1}$. We take $y \in Y^{1}$. Then, we obtain the following commutative diagram of inclusions

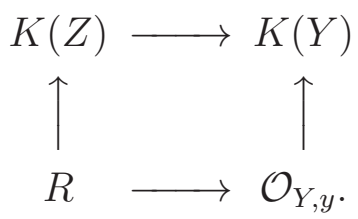

Take a normal projective compactification $Z \subset \bar{Z}$ and the normalization of a blowup of the indeterminacy of $Y \rightarrow \bar{Z}$, denoted by 
$\pi^{\prime}: Y^{\prime} \rightarrow \bar{Z}$. We obtain

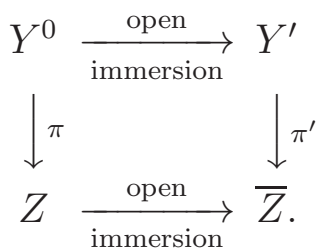

Fix a point $y^{\prime}$ over $y$. Then, we obtain $R \subset \mathcal{O}_{Y^{\prime}, y^{\prime}}$. Therefore, $\pi^{\prime}\left(y^{\prime}\right)$ is a point $z$ in $Z=\operatorname{Spec} R$. It suffices to show that $\pi^{-1}(z)=\pi^{\prime-1}(z)$. Note that $\pi^{-1}(z)$ is proper over $k$ and that $\pi^{-1}(z)=\pi^{\prime-1}(z) \cap Y^{0}$ is an open subset of $\pi^{\prime-1}(z)$. Since $\pi_{*} \mathcal{O}_{Y^{0}}=\mathcal{O}_{Z}$, the field $K(Z)$ is algebraically closed in $K(Y)$. Therefore, $\pi_{*}^{\prime} \mathcal{O}_{Y^{\prime}}=\mathcal{O}_{\bar{Z}}$. In particular, $\pi^{\prime-1}(z)$ is connected, which implies $\pi^{-1}(z)=\pi^{\prime-1}(z)$. Indeed, otherwise $\pi^{-1}(z)=\pi^{-1}(z) \cap Y^{0}$ is not proper over $k$.

We prove the main result of this section.

Proposition A.4. Let $k \subset k^{\prime}$ be a field extension. Let $X$ be a proper geometrically normal and geometrically connected variety over $k$. Let $X \supset X^{0} \rightarrow V$ and $X \otimes_{k} k^{\prime} \supset Y^{0} \rightarrow W$ be MRCC fibrations of $X$ and $X \otimes_{k} k^{\prime}$, respectively. Then $\operatorname{dim} V=\operatorname{dim} W$.

Proof. For a proper variety $V$, set $r(V):=\operatorname{dim} V-\operatorname{dim} W$ where $V \supset$ $V^{0} \rightarrow W$ is an MRCC fibration. Note that $r(V)$ is well-defined because $W$ and $W_{1}$ are birational for another MRCC fibration $V \supset V_{1}^{0} \rightarrow W_{1}$. It is enough to show $r(X)=r\left(X \otimes_{k} k^{\prime}\right)$.

We show $r(X) \leq r\left(X \otimes_{k} k^{\prime}\right)$. Take an RCC fibration $X \supset X^{0} \rightarrow Z$ such that the dimension of a general fiber is $r(X)$. Then, taking the base change to $k^{\prime}$, we obtain an RCC fibration $X \otimes_{k} k^{\prime} \supset X^{0} \otimes_{k} k^{\prime} \rightarrow$ $Z \otimes_{k} k^{\prime}$. Therefore $r(X) \leq r\left(X \otimes_{k} k^{\prime}\right)$.

It suffices to prove $r(X) \geq r\left(X \times_{k} k^{\prime}\right)$. We obtain an RCC fibration $X \otimes_{k} k^{\prime} \supset Y^{0} \rightarrow Z$ such that $\operatorname{dim} X-\operatorname{dim} Z=r\left(X \otimes_{k} k^{\prime}\right)$.

We prove that we may assume that $\left[k^{\prime}: k\right]<\infty$. We obtain a family of RCC fibrations $X \otimes_{k} R \supset Y_{R}^{0} \rightarrow Z_{R}$ over some intermediate ring $k \subset R \subset k^{\prime}$ which is of finite type over $k$. Taking a general closed point of $\operatorname{Spec} R$, we may assume $\left[k^{\prime}: k\right]<\infty$.

If $k^{\prime} / k$ is purely inseparable, then the assertion can be easily proved. Thus, we may assume that $k^{\prime} / k$ is a finite separable extension. Moreover, by taking the Galois closure of $k^{\prime} / k$, we may assume that $k^{\prime} / k$ is a finite Galois extension.

Assume that $k^{\prime} / k$ is a finite Galois extension. We have an MRCC fibration $Y:=X \otimes_{k} k^{\prime} \supset Y^{0} \rightarrow Z$ such that $\operatorname{dim} Y-\operatorname{dim} Z=r\left(X \otimes_{k} k^{\prime}\right)$. 
Since $k^{\prime} / k$ is a Galois extension, so is $K(Y)=K\left(X \otimes_{k} k^{\prime}\right) / K(X)$. Set

$$
G:=\operatorname{Gal}(K(Y) / K(X))=\left\{\sigma_{1}, \cdots, \sigma_{N}\right\}
$$

By shrinking $Z$, we may assume $Z=\operatorname{Spec} R_{Z}$. We fix an embedding $K(Z) \subset K(Y)$. Let $\sigma_{i}^{*}: Y \rightarrow Y$ be the induced automorphism.

We show that $K(Z)=\sigma_{i}(K(Z))$ for every integer $1 \leq i \leq N$. Fix $1 \leq i \leq N$. We obtain another MRCC fibration

$$
Y=\sigma_{i}^{*}(Y) \supset \sigma_{i}^{*}\left(Y^{0}\right) \rightarrow \operatorname{Spec} \sigma_{i}\left(R_{Z}\right) .
$$

Then, we see that $\operatorname{Spec} R_{Z}$ and $\operatorname{Spec} \sigma_{i}\left(R_{Z}\right)$ are birational. This implies $K(Z)=\sigma_{i}(K(Z))$.

Thus, each $\sigma_{i}$ induces an birational automorphism $\sigma_{i}: Z \rightarrow Z$. Since $G=\left\{\sigma_{1}, \cdots, \sigma_{N}\right\}$ is a finite group, we can find a non-empty open subset $Z^{\prime} \subset Z$ such that the induced rational map $Z^{\prime} \rightarrow Z^{\prime}$ is an isomorphism, i.e., automorphism. By replacing $Z$ with $Z^{\prime}$, we may assume that $\sigma_{i}: Z \rightarrow Z$ is an automorphism. In particular $\sigma_{i}\left(R_{Z}\right)=R_{Z}$

We show $Y^{0}=\sigma_{i}^{*}\left(Y^{0}\right)$ for every $i$. By Lemma A.3, we obtain

$$
Y^{0}=\left\{y \in Y \mid R_{Z} \subset \mathcal{O}_{Y, y}\right\} .
$$

Therefore,

$$
\begin{aligned}
\sigma_{i}^{*}\left(Y^{0}\right) & =\sigma_{i}^{*}\left(\left\{y \in Y \mid R_{Z} \subset \mathcal{O}_{Y, y}\right\}\right) \\
& =\left\{y^{\prime}:=\sigma_{i}^{*}(y) \in Y \mid R_{Z} \subset \mathcal{O}_{Y, y}\right\} \\
& =\left\{y^{\prime} \in Y \mid R_{Z} \subset \mathcal{O}_{Y,\left(\sigma_{i}^{*}\right)^{-1}\left(y^{\prime}\right)}\right\} \\
& =\left\{y^{\prime} \in Y \mid R_{Z} \subset \sigma_{i}^{-1}\left(\mathcal{O}_{Y, y^{\prime}}\right)\right\} \\
& =\left\{y^{\prime} \in Y \mid \sigma_{i}\left(R_{Z}\right) \subset \mathcal{O}_{Y, y^{\prime}}\right\} \\
& =\left\{y^{\prime} \in Y \mid R_{Z} \subset \mathcal{O}_{Y, y^{\prime}}\right\} \\
& =Y^{0}
\end{aligned}
$$

Set $X^{0}:=\beta\left(Y^{0}\right)$, where $\beta: Y \rightarrow X$. Then, by $Y^{0}=\sigma_{i}\left(Y^{0}\right)$, we obtain

$$
\beta^{-1}\left(X^{0}\right)=\bigcup_{1 \leq i \leq N} \sigma_{i}\left(Y^{0}\right)=\bigcup_{1 \leq i \leq N} Y^{0}=Y^{0} .
$$

By Lemma A.1, we obtain the following commutative diagram

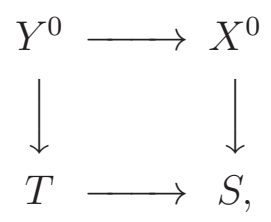

where $S$ is an affine normal $k$-variety, $T \rightarrow S$ is a finite surjective morphism, and $X^{0} \rightarrow S$ is a proper surjective morphism. Since fibers of 
$Y^{0} \rightarrow T$ are RCC, so are the fibers of $X^{0} \rightarrow S$. Therefore $X \supset X^{0} \rightarrow S$ is an RCC fibration, which implies

$$
r(X) \geq \operatorname{dim} X-\operatorname{dim} S=\operatorname{dim} Y-\operatorname{dim} T=r(Y)=r\left(X \otimes_{k} k^{\prime}\right) .
$$

\section{REFERENCES}

[Bad01] L. Bădescu, Algebraic Surfaces, Universitext, Springer-Verlag, New York, 2001.

[Bir] C. Birkar, Existence of flips and minimal models for 3-folds in char p, to appear in Ann. Sci. de l'École Norm. Sup.

[Bou03] N. Bourbaki, Elements of Mathematics: Algebra II: Chapter 4-7, Springer Verlag, 2003.

[CTX] P. Cascini, H. Tanaka, C. Xu, On base point freeness in positive characteristic, to appear in Ann. Sci. de l'École Norm. Sup.

[CZ] Y. Chen, L. Zhang, The Subadditivity of the Kodaira Dimension for Fibrations of Relative Dimension One in Positive Characteristics, to appear in Math. Research Letters.

[Con00] B. Conrad, Grothendieck Duality and Base Change Lecture Notes in Math., Vol. 1750, Springer, Berlin 2000.

[Getal15] Y. Gongyo, Z. Li, Z. Patakfalvi, K. Schwede, H. Tanaka, H. R. Zong, On rational connectedness of globally F-regular threefolds, Advances in Math., Vol. 280 (2015), 47-78.

[HM07] C. D. Hacon, J. MKernan, Shokurov's Rational Connectedness Conjecture, Duke Math. J., Vol. 138, Number 1 (2007), 119-136.

[Har66] R. Hartshorne, Residues and Duality, Lecture Note in Math. 20 (1966), Springer-Verlag, Berlin-New York.

[Kee99] S. Keel, Basepoint freeness for nef and big linebundles in positive characteristic, Ann. Math, 149 (1999), 253-286.

[Kol96] J. Kollár, Rational curves on algebraic varieties, Ergebnisse der Mathematik und ihrer Grenzgebiete. 3. Folge. A Series of Modern Surveys in Mathematics [Results in Mathematics and Related Areas. 3rd Series. A Series of Modern Surveys in Mathematics], Springer-Verlag, Berlin, 1996.

[Kol13] J. Kollár, Singularities of the minimal model program, Cambrigde Tracts in Mathematics, Vol. 200, 2013.

[KMM92] J. Kollár, Y. Miyaoka, S. Mori, Rational connectedness and boundedness of Fano manifolds, J. Diff. Geom., Vol. 36 (1992), 765-779.

[KM98] J. Kollár, S. Mori, Birational geometry of algebraic varieties, Cambrigde Tracts in Mathematics, Vol. 134, 1998.

[LH09] J. Lipman, M. Hashimoto, Foundation of Grothendieck Duality for Diagrams of Schemes, Lecture Notes in Math., Vol. 1960, Springer-Verlag, Berlin (2009).

[Mor82] S. Mori, Threefolds whose canonical bundles are not numerically effective, Ann. of Math., Vol. 116, No. 1 (1982), 133-176.

[Rei94] M. Reid, Nonnormal del Pezzo surfaces, Publ. RIMS, Kyoto Univ., 30, 1994, 695-727. 
[Sch09] S. Schröer, On genus change in algebraic curves over imperfect fields, roc. Amer. Math. Soc. 137 (2009), 1239-1243.

[Sch10] S. Schröer, On fibrations whose geometric fibers are nonreduced, Nagoya Math. J. 200 (2010), 35-57.

[Tan14] H. Tanaka, Minimal models and abundance for positive characteristic log surfaces, Nagoya Math. Journal, Vol. 216 (2014), 1-70.

[Tat52] J. Tate, Genus change in inseparable extension of function fields, Proc. Amer. Math. Soc., 3 (1952), 400-406.

[Zha06] Q. Zhang, Rational connectedness of log Q-Fano varieties, J. Reine Angrew. Math., 590 (2006), 131-142.

Department of Mathematics, Imperial College, London, 180 Queen's Gate, London SW7 2AZ, UK

E-mail address: h.tanaka@imperial.ac.uk 